The Neurocognitive Bases of Numerical Cognition

Francesco Sella, Charlotte Hartwright, \& Roi Cohen Kadosh University of Oxford

Department of Experimental Psychology, University of Oxford, Oxford, OX1 3UD, UK

This preprint outlines a chapter on the subject of 'Numerical Cognition' for inclusion in the forthcoming work tentatively entitled Stevens' Handbook of Experimental Psychology, Fourth Edition, Volume Three: Language \& Thought (the "Work"), authored/edited by Sharon L. Thompson-Schill, published by JOHN WILEY \& SONS 


\begin{abstract}
Numerical Cognition describes the processes that one uses to assimilate, ascribe and manipulate numerical information. This chapter is organised into two sections. The first draws heavily on data from Developmental and Experimental Psychology. We use this to outline core findings related to processing numerical information in humans. In particular, we describe the trajectory of the acquisition of basic numerical skills. Starting in early infancy, we outline the processes that are believed to underlie non-symbolic representation. Next, we summarise core studies that examine the representation of symbolic quantities (Arabic system). Lastly, we briefly report the relationship between basic numerical processing and mathematical achievement. The second part of the chapter explores evidence from Neuropsychology and Neuroscience. The core methodological approaches used are briefly outlined with sign-posting to relevant literature. Next, we examine data from early lesion studies, followed by a short review of one of the most influential models in the study of Numerical Cognition, the Triple Code Model. Lastly, we look at the neurocognitive features of number, such as different modes of representation and the processing of quantity. Throughout, the core literature plus recent advances are summarised, giving the reader a thorough grounding in the Neurocognitive Bases of Numerical Cognition.
\end{abstract}




\section{The Neurocognitive Bases of Numerical Cognition}

The ability to process numerical information is crucial to navigate the human world. An understanding of numerical quantity is informative to decisions regarding basic survival, for example, to enable one to choose the habitat with the most plentiful supply of food and mates, whilst minimising the risk of encountering predators. Infants and animals have been suggested to have the ability to process non-symbolic numerical quantities as represented by comparing sets of objects. Beyond this, humans have devised culturally meaningful symbols, such as Arabic numerals, on which quantities can be represented in a symbolic way. Both symbolic and non-symbolic processing of numerical information has been widely investigated within Experimental Psychology and Cognitive Neuroscience. In this chapter, we review some of the core findings regarding numerical cognition. First, we review the main results relating to the development of numerical skills, from non-symbolic to symbolic representations. We present those paradigms that are most commonly used to study the acquisition of numerical skills in infants and young children, and show how the representation of numerical information changes over time. We further describe those systems that are considered maturationally complete in adults and how behavioural data can be informative to theories regarding the processes that underpin numerical cognition. Next, we briefly describe the link between numerical cognition and mathematical achievement, and how basic numerical processes may support the acquisition of advanced mathematical procedures and concepts. In the latter part of this chapter, we review evidence from neuroimaging and neuropsychology to provide a neurocognitive account of numerical cognition. We summarise a core model, the Triple Code Model, which has stimulated numerous functional neuroimaging studies. We also examine the neural bases of symbolic and non-symbolic number and core systems that represent quantity and estimation. The primary aim of this chapter is to give the reader an overview of the main neurocognitive 
features of number processing, from infant- to adult-hood. Though not exhaustive, we set out to cover those aspects that we consider to be the most investigated within the field, with primary focus on numerical, as opposed to mathematical, cognition. For an extensive overview of the literature relating to numerical and mathematical cognition, we recommend Cohen Kadosh and Dowker (2015).

\section{InSIGHTS FROM DEVELOPMENTAL AND EXPERIMENTAL PSYCHOLOGY}

\section{Pre-verbal numerical mechanisms}

In the last thirty years, several studies have established that humans (and other animal species) are born equipped with basic numerical mechanisms to process numerical quantities. Two basic pre-verbal mechanisms have been individuated: The Object Tracking System (OTS; or Object file system) and the Approximate Number System (ANS; Feigenson, Dehaene, \& Spelke, 2004; Piazza, 2010). The former is considered a domain-general mechanism to track the spatio-temporal characteristics of a limited number of items (approximately 3-4), which are assigned with a visual index. The Object Tracking System allows individuals to rapidly and accurately determine the numerosity of small sets without using counting routine, a phenomenon called subitizing (Mandler \& Shebo 1982; Kaufman et al. 1949; Trick \& Pylyshyn 1994; Pylyshyn 2001). The crucial signature of the Object Tracking System is its limited storage capacity (i.e., 3-4 elements), which mimics that of visual short term memory (VSTM). For this reason, some authors have suggested the OTS is intimately linked to VSTM (Piazza et al. 2011; Knops, Piazza, et al. 2014). In the Approximate Number System, instead, each numerosity is represented as a Gaussian curve of activation on a metaphorical mental number line. There are two main competitive mathematical models that formally describe the Approximate Number System: In the linear model (Gallistel \& Gelman, 2000; Gallistel \& Gelman, 1992; Whalen, Gallistel, \& Gelman, 1999), the curves of activation are linearly spaced with an increasing standard deviation (i.e., 
scalar variability) as a function of numerical magnitude. Conversely, in the logarithmic model (Dehaene 2003), the standard deviation of the Gaussian curves is constant, whereas the distance between numerical magnitude is logarithmically compressed (Figure 1). Despite the differences in their formulation, both models explain behavioural results and make similar predictions (but see Dehaene, 2003). The characteristic signature of the Approximate Number System is the ratio-dependent effect, which states that the discriminability between two numerical sets crucially depends on their numerical ratio, thereby obeying the Weber law. The more the numerical ratio (i.e., smaller set/larger set) approaches one, the harder the discrimination is. Conversely, when the numerical ratio approaches zero, identifying the larger between two numerical sets becomes an easy task.

\section{$<$ FIGURE 1 ABOUT HERE $>$}

<FIGURE FROM: Feigenson, Dehaene, \& Spelke, 2004>

\section{Numerical skills in infants}

\section{Small numerical quantities}

Studies using infants allow researchers to investigate core human cognitive systems (Spelke 2000) when the influence of cultural or educational factors is limited or even absent. In order to investigate numerical representations in infants, many studies have employed the habituation-dishabituation paradigm (Oakes 2011; Fantz 1964). This technique relies on the fact that infants display longer looking times for novel stimuli in their environment. In the habituation phase, the same stimulus is repeatedly presented causing a progressive decrease of the child's looking times. In the dishabituation phase, when a novel stimulus is shown, looking times suddenly increase if the child has perceived the difference between the familiar and the novel stimulus. Conversely, looking times continue decreasing when a child fails to detect any difference between previously presented stimuli and the novel ones. In a 
pioneering study on numerical cognition, different images representing sets composed of two dots were repeatedly presented to 22 week-old infants until they habituated to this specific numerosity. Thereafter, a stream of novel images representing a set with three dots was presented to children. Infants' looking times were longer immediately after the presentation of sets composed of three dots, thereby suggesting that infants perceived a change in numerosity between familiar and novel sets (Starkey \& Cooper 1980). The physical characteristics of the presented sets (e.g., length array, density, and dots position) were manipulated to prevent infants basing their discrimination systematically on a fixed nonnumerical feature of the sets. Moreover, the order of presentation was also reversed ( 3 vs. 2 dots) to verify that infants actually dishabituated to numerosity rather than the complexity of the sets. Infants reliably discriminated 2 vs. 3 dots (or 3 vs. 2) but they failed to dishabituate when comparing 4 vs. 6 dots (and 6 vs. 4). The same pattern of results was replicated in a sample of new-born infants, aged around 55 hours old (Antell \& Keating 1983). Similar findings were obtained with 10 to 12 month-old infants who dishabituated in the comparison of 2 vs. 3 items but failed with 4 vs. 5 comparison (Strauss \& Curtis 1981). However, one study found that 4-month-old infants can discriminate 4 vs. 5 items even when several nonnumerical features of the presented sets were controlled (Treiber \& Wilcox 1984).

Remarkably, infants can attend to the numerosity of the presented sets even when the item type (e.g., dogs, houses), position, and size were varied across trials. Van Loosbroek and Smitsman (1990) found that infants from 5 months of age can dishabituate also to moving stimuli, thereby highlighting that children can focus on numerosity instead of relying on visual patterns of the stimuli (for similar results, Wynn, Bloom, \& Chiang, 2002). Despite these findings, some studies have suggested that infants actually dishabituate to change in physical variables of the set (e.g., contour length, surface area) rather than numerosity (Clearfield \& Mix 1999; Clearfield \& Mix 2001). 
In summary, infants can reliably discriminate between small numerical sets within the Object Tracking System capacity limit, whereas they fail to dishabituate with comparisons involving larger numerosities. Generally speaking, it remains unclear whether infants can discriminate small numerical quantities or their numerical processing is heavily influenced by non-numerical features (Feigenson, Carey \& Hauser 2002; Feigenson, Carey \& Spelke 2002).

\section{Large numerical quantities}

The ability to discriminate between numerical quantities goes beyond the capacity of the Object Tracking System to encompass larger sets. In their seminal study, Xu and Spelke (2000) habituated 6-months old children to a stream of images displaying 8 dots, whose nonnumerical physical characteristics (e.g., perimeter, density) were continuously varied.

Thereafter, an image with 16 or 12 dots was presented and children's looking times were recorded. Children showed longer looking times for 16 dots but not for 12 dots. The same pattern of results was observed when infants were initially presented with 16 dots in the habituation phase and eight dots in the dishabituation phase. Infants can reliably discriminate between numerical quantities that exceed the Object Tracking System capacity limits as long as the ratio between the presented numerical sets is far from 1, and their ability will depend on their age and numerical competence (see below). Their data indicated that at 6 months of age, children can reliably discriminate numerosities in a ratio of 1:2 (8 vs. 16) but fail with the ratio of 2:3 (8 vs. 12). The same ratio dependent effect was found when 6 months-old infants were presented with even larger numerosities (i.e., 16 vs. 32 and 16 vs. 24; Xu, Spelke, \& Goddard, 2005), suggesting the crucial role of the ratio between the presented numerosities, rather than the numerical distance, between sets. The ability to discriminate between numerical quantities, also called number acuity, increase as a function of age. At 10 months of age, infants can reliably discriminate numerical sets with a numerical ratio of 2:3 (i.e., 8 vs. 12 elements), but still fail with 4:5 (i.e., 8 vs 10 elements). Infants' numerical skills have been widely explored in the visual domain, where similar pattern of results are found 
when infants enumerate sounds and actions in a scene (Wood \& Spelke 2005; Sharon \& Wynn 1998). This finding is supported by other studies, which found that infants can match numerical quantities across different modalities, for example in the auditory or visual domain, suggesting that numerical representation is abstract (Izard et al. 2009; Starkey et al. 1983), although whether such findings support abstract numerical representation has been questioned (for a comprehensive review see, Cohen Kadosh \& Walsh 2009). A vast internetbased study demonstrated that number acuity dramatically improves across life-span, with peak performance in the late twenties, followed by a slow decline in later life (Halberda et al. 2012).

One of the crucial points regarding the comparison of non-symbolic numerical quantities is whether individuals can base their discrimination on numerical information. The effect of physical characteristics of stimuli on non-symbolic numerical processing is a recurring issue in the field (Gebuis et al. 2014; Gebuis \& Reynvoet 2012b). Density, perimeter, item size, area, luminance are physical features that covary with the numerosity of a given numerical set and controlling for all sensory cues in a display will lead to two equal numerical quantities. For this reason, it is necessary to create numerical sets in which the influence of physical variables is minimize or can be taken into account (Gebuis \& Reynvoet 2012a). This procedure ensures that individuals are actually extracting numerical information, rather than relying on non-numerical features to accomplish a discrimination task. However, it has been suggested that most, if not all, studies in the field that conclude that non-symbolic numbers were processed had a limitation in their design (Gevers et al., 2016). Based on that several researchers in the field have put into question whether the results so far in the field of numerical cognition from infants, as well as animals and adults reflect numerical processing, rather than non-numerical sensory cues (Gebuis et al. 2016). 
Infants' numerical skills go beyond simple discrimination to include arithmetical operations on non-symbolic quantities. In her seminal study, Wynn (1992a) used the violation of expectation paradigm to investigate whether infants can perform non-symbolic addition and subtraction. The violation of expectation paradigm is based on the premise that infants look longer to events that are physically impossible. Young children have expectations about the physical world and their attention is captured when these expectations are violated (Sirois \& Mareschal 2002). Wynn presented 5 months-old infants with a doll whose view was subsequently occluded by a panel. Thereafter, the experimenter placed a second doll next to the first. When the occluding panel was removed, infants could see either one or two dolls. Children looked longer when only one doll was displayed because this outcome violated their expectation about the addition operation. Similarly, in the subtraction condition, two dolls were initially presented and then occluded with the panel. Children saw a hand removing one doll from behind the panel and then the panel was removed. Again, infants looked longer when two dolls were still present despite the subtractive action. Infants display specific expectations related to arithmetical operations. These findings have been repeatedly tested to verify whether infants respond to other characteristics of the stimuli such as the invariability of the paradigm outcome (Wynn 1992a), the identity of the dolls (Simon et al. 1995), or the spatial location of the objects (Koechlin, 1997; for a detailed review see, McCrink \& Birdsall, 2015), and has been linked to neurophysiological measures in the infant brain (Berger, Tzur, \& Posner, 2006). Despite the robustness of the finding, it remains unclear whether infants truly possess arithmetical expectation or the observed results are a consequence of children's ability to track spatiotemporal characteristics of the objects. McCrink and Wynn (2004) have shown that nine-month-old infants can reliably detect the wrong outcome of additions and subtractions with larger numerosities $(5+5=10$ or $5 ; 10-5=5$ or 10$)$, when controlling for nonnumerical physical characteristics. For both addition and subtraction, children looked longer at incorrect compare to correct outcomes. The same pattern of results was found with larger 
numerosities $(6+4=5$ or 10 or $20 ; 14-4=5$ or 10 or 20 ; McCrink \& Wynn, 2009), along with a tendency to overestimate the result of non-symbolic addition and underestimate the result of subtraction, an effect called operational-momentum, which has been repeatedly found in adults (Pinhas \& Fischer 2008; Knops, Dehaene, et al. 2014; McCrink et al. 2007). Infants can also perceive difference in numerical ratio of visual sets. McCrink and Wynn (2007) habituated six-months-old infants to a series of slides representing two different sets which varied in numerosity but with a constant ratio (4 vs. 8,10 vs. 20). As with other studies, physical non-numerical characteristics of the numerical sets were strictly controlled to prevent children from using non-numerical information. Infants habituated to 1:2 ratio showed longer looking time when the 1:4 ratio was presented. The same pattern of results was found when infants were habituated to the 1:4 ratio, and the 1:2 ratio was shown in the dishabituation phase. Children failed to dishabituate when a closer ratio comparison was presented 1:2 ratio vs. 1:3 ratio. Therefore, the representation of numerical ratios displays the typical Approximate Number System signature, where difficulty in discrimination varies as a function of numerical ratio (i.e., ratio-dependent effect).

In sum, infants demonstrate the ability to discriminate between small and large numerical quantities as well as performing arithmetical operations and ratio comparisons. The discrimination of small quantities display the classic Object Tracking System capacity limit and seems to be more influenced by non-numerical characteristics of the stimuli (e.g., item size). This influence might be connected to the fact that the Object Tracking System creates a specific object-file including spatio-temporal characteristics of each memorized stimulus (Feigenson, Carey \& Spelke 2002). The comparison of larger quantities and ratios rely on the Approximate Number System, as indicated by the presence of the ratio-dependent effect. However, there is on-going debate as to whether people base discrimination comparisons on non-numerical characteristics of the stimuli (Clearfield \& Mix 1999; Clearfield \& Mix 2001; Gebuis et al. 2014). 


\section{From approximate non-symbolic to exact symbolic representation of numbers: The development of counting skills.}

The acquisition of counting skills represents the first connection between pre-verbal numerical mechanisms and a culturally-determined symbolic system. From two years of age toddlers begin to implement counting routines to correctly enumerate sets of objects in their environment (Wynn 1992b). The repeated connection between physical elements and the corresponding number-words contributes to the establishment of a robust mapping between non-symbolic and symbolic representation of numerical quantities. This procedure is similar to any other mapping between a word and its specific meaning during the extension of personal vocabulary. Nevertheless, number-words represent a particular case because they refer to the property of a set rather than to a characteristic of a specific object (Wynn 1992b). According to the "Counting Principles" theory (Gelman \& Gallistel 1978), children possess innate counting principles that guide them in recognizing counting as an activity to determine the numerosity of a given set (Gelman \& Gallistel 1978; Wynn 1990). For accomplished counting, children must respect three basic principles. They have to: i) recite the numberword sequence in the established order (stable order principle); match each object in the set to one, and only one, number-word (one-to-one correspondence principle); identify that the last number-word represents the numerosity of the set (cardinality principle). There are other two additional principles: the abstraction principle states that any collection of objects can be counted (e.g., apples, ideas, dreams etc.); and the order-irrelevance principle, which states that the order in which the items are counted is irrelevant. The achievement of counting principles is a long and error prone process that engages children for about 1 year, usually between 2 and 4 years of age. The counting principle theory strongly underlines an initial role of these principles in shaping the acquisition of counting skills (principle first view).

Conversely, other theories have emphasised that, initially, counting is a routine through which children later acquire the counting principles (principle after view). From this 
perspective, counting is first an imitation activity shaped by parents, teachers and peers (Briars \& Siegler 1984; Fuson 1988), and only later children learn that counting specifically refers to the determination of numerosity of a given set. According to the "Different Contexts" theory (Fuson 1988), number-words assume different meanings depending on the context. In the sequence context, children can recite number-words aloud in the correct order because they memorized it as unique sequence of sounds without any specific numerical meaning. In the counting context, children can actually segment the numerical sequence and assign a number word to only one counted object (one-to-one correspondence). Nevertheless, children still lack the understanding that the last number word represents the numerosity of the counted set (Cardinal context).

An interesting aspect of counting is describing how children progressively acquire the cardinality principle and how they extend it to the counting list. According to the Knowerlevel theory (Wynn 1990; Carey 2001; Sarnecka \& Carey 2008), children's acquisition of the cardinal meaning of number-words follows predictable developmental stages. Initially, children are considered pre-numerical knowers because they lack any numerical meaning of the number-words. When requested to provide a certain number of objects (as in the Give-aNumber task; Wynn, 1990), children usually collect a handful of items without implementing any counting strategy. Subsequently, children learn the cardinal meaning of the number-word "one"; therefore, they can correctly provide one item when it is requested. However, oneknowers still fail with larger numerosities. Interestingly, one-knowers do not bring one item when larger numerosities are requested because they know the cardinal meaning of the number-word “one”. Similarly, 2-, 3-, and 4-knowers correctly provide 2, 3, and 4 items respectively, but they still fail with larger numerosities. These children are usually defined as Subset-knowers because their cardinal meaning of number-words is limited to a subset of the counting list (i.e., from 1 to 4; Le Corre, Van de Walle, Brannon, \& Carey, 2006). This limit to 3-4 four elements matches the number of items that can be distinctively perceived without 
counting by means of subitizing (Object Tracking System capacity limit; Klahr \& Wallace, 1973). Successively, children crucially realize that the next number-word in the counting list corresponds to one additional element in the counted set (i.e., successor function, Gelman \& Galistell, 1978). Children at this stage can extend the cardinal principle to the whole counting list, thereby becoming Cardinal-Principle knowers. With the full acquisition of cardinality principle, children understand that the last recited number-word identifies the numerosity of the counted set. It has been suggested that learning to count resembles a conceptual bootstrapping process (Carey 2009). Initially, by respecting the counting rules (e.g., fixed order of number-words, one-to-one correspondence), children create a structure in which number-words are simply placeholders with specific relations between each other (e.g., "two" comes after "one") but they still lack a numerical meaning. Only later, through a conceptual bootstrapping process, do children progressively fill the placeholders with numerical meaning. It is worth noting that the presence of knower-levels has been replicated across different countries (Sarnecka, Kamenskaya, Yamana, Ogura, \& Yudovina, 2007), thereby suggesting the robustness of the theory despite the differences between cultures and languages.

In summary, children use counting to create a reliable association between objective numerosities and symbolic (e.g., Arabic numbers in the western culture) representation of numerical quantities. The initial counting routine progressively leads children to understand that the last pronounced number-word corresponds to the cardinality of the set. The acquisition of the cardinality principle is a crucial mile stone in numerical development because it allows one to assign numerical meaning to culturally-defined and totally arbitrary symbols. 


\section{Non-symbolic and symbolic numerical processing in children and adults}

\section{Subitizing}

Studies with infants have employed habituation paradigms to demonstrate that young children can discriminate between small numerical quantities. Different experimental paradigms have been implemented to assess the same ability in older children and adults. Typically, individuals are asked to enumerate the items in a visually presented set. When few elements are displayed (1-4), the accuracy is high and reaction times (RTs) fast, a phenomenon called subitizing (Kaufman et al., 1949; Trick \& Pylyshyn, 1994). Subitizing seems to rely heavily on attentional and visual short term memory capacity, which allows participants to track the spatiotemporal characteristics of a few items (Burr et al. 2010; Piazza et al. 2011; Piazza 2010). When the number of items increases beyond 4, individuals can rely on estimation or counting, depending on the time at their disposal. When the items are presented for a short time, individuals can only estimate the presented numerosity. In this case, reaction times increase with numerosity to a point, and then reach a plateau (i.e., RTs remain constant despite the increase in displayed numerosity), whereas the accuracy is markedly lower compare to subitizing. On the other hand, with unlimited time, individuals can count the items, thereby increasing response time as a function of numerosity, while accuracy remains high. Several indexes may be used to obtain a reliable measure of subitizing and counting skills, such as the RTs slopes separately for subitizing and counting range, or the estimation of subitizing range as the discontinuity point in RT slopes (Reeve, Reynolds, Humberstone, \& Butterworth, 2012; Schleifer \& Landerl, 2011; for an assessment based on accuracy see, Sella, Lanfranchi, \& Zorzi, 2013; Trick \& Pylyshyn, 1994). However, when estimating subitizing capacity, these paradigms require individuals to determine the numerosity of a set and also to retrieve its verbal label. To avoid the influence of verbal processing on subitizing, Sella and colleagues (Sella et al. 2013) adopted a match-to-sample task in which a sample set ranging from 1 to 9 was briefly presented, followed by a target set 
whose numerosity differed for one element $(-1$ or +1$)$. Participants had to determine whether the two sets had the same or different numerosity. The accuracy in comparing numerical sets around the Object Tracking System capacity (i.e., 3 vs. 4, 4 vs. 5) represented a valid and pure assessment of the subitizing limit without involving any verbal component.

\section{Numerosity estimation}

In an exhaustive study, Izard and Dehaene (2008) asked participants to verbally estimate the numerosity of visually presented sets of dots. Both the mean and the standard deviation of numerical estimates increased as a function of target numerosities, thereby suggesting the presence of progressive noise in the representation of numerical quantities (see the manuscript for a detailed theoretical model of the numerical estimation). Moreover, individuals tended to regularly provide estimates that were lower compared target numerosities. For example, numerical sets comprising dots ranging from 60 to 82 were estimated as composed of only 30 dots. However, this pattern of underestimation could be rectified through a calibration procedure in which participants were shown a numerical set and informed about its real numerosity. The calibrated numerosity works as a reference to correct subsequent estimates, which were more accurate but still characterized by scalar variability. Notably, a calibration procedure can induce patterns of overestimation and underestimation by deceptively informing participants about the number of items in the calibration set. For instance, a calibration set composed of 30 elements can be presented to participants as it was composed of 20 elements, thereby inducing a pattern of underestimation. Conversely, a pattern of overestimation can be induced when participants are told that the calibration set with 30 elements is actually composed of 40 elements. Interestingly, the effect of (mis-)calibration was found only for numerosities larger than 1215 in adults, and larger than 6 in children. For smaller numerosities, individuals adopted a direct mapping which was insensitive to the (mis-)calibration procedure (Sullivan \& Barner 2014). 


\section{Numerosity production and re-production}

The vast majority of research has focused on the mapping from non-symbolic numerical quantities to symbolic numbers. However, some studies have also explored the opposite mapping, from symbolic numbers to non-symbolic numerosities, namely, numerical production. In numerical production paradigms, participants are usually asked to increase (or decrease) a given non-symbolic numerosity to reach a symbolic target number. For instance, participants are instructed to produce a set comprised of 30 dots (i.e., the target number). A set comprised of few dots is initially presented on the screen and participants repeatedly press a keyboard button to increase the number of dots to reach the target number. In the accomplishment of this task, individuals cannot use counting strategies. Both animals' and humans' estimates are characterized by scalar variability, as a signature of the reliance on the Approximate Number System when generating a numerical quantity (Platt \& Johnson, 1971; Whalen, Gallistel, \& Gelman, 1999). Similarly, pre-counting children rely on the Approximate Number System when spontaneously encoding numerical information in their environment. In a recent study (Sella et al. 2015a), children watched an experimenter inserting 'food tokens' into an opaque animal puppet and then were asked to replicate the puppet-feeding behaviour. Children's response distributions were centred on the target numerosities with an increasing (scalar) variability, in agreement with the deploying of the Approximate Number System.

Consistent with the bidirectional mapping hypothesis (Castronovo \& Seron 2007), individuals underestimate visually presented numerosity and overestimate numerosity in numerical production. Indeed, the underestimation of visually presented numerical quantities consequently leads to the production of larger sets when asked to match a given number. For example, if 70 items are perceived as "thirty" in a numerical estimation task (i.e., underestimation), therefore individuals tend to create a set with approximately 70 items to match "thirty" in a numerical production task (i.e., overestimation). Conversely, the pattern 
of individuals' estimates are negligibly affected by under- and over-estimation patterns when a non-symbolic numerosity of a given set has to been increased or decreased to match the non-symbolic numerosity of a target set (i.e., numerical re-production; Crollen, Castronovo, \& Seron, 2011). Recently, Ebersbach and Erz (2014) investigated numerical estimation, numerical production, and numerical reproduction skills in a sample of preschoolers, first graders, third graders and adults. Consistent with the bidirectional mapping hypothesis, adults and older children displayed a pattern of underestimation in the numerical estimation task and a pattern of overestimation in the numerical production task. Conversely, younger children (i.e., preschoolers and first graders) displayed less straightforward results, which were conceivably due to their poor familiarity with the presented numbers. In short, numerical estimation and production appears to rely on the Approximate Number System and can be characterized by patterns of under- and overestimation.

Non-symbolic number comparison: Number acuity

Earlier in this chapter, we outlined that infants are born equipped with the ability to discriminate between large numerical quantities. When assessing the same ability in older children and adults, two numerical sets are usually presented respectively on the left and the right side of the screen for a brief period and individuals are asked to choose the set with the larger (or the smaller) numerosity (Figure 2). Numerical sets are usually comprised of dots (or other geometrical shapes), which can be presented side-by-side, sequentially, or intermixed using different colours for the two sets (for a comparison of different presentation modalities see, Price, Palmer, Battista, \& Ansari, 2012). 


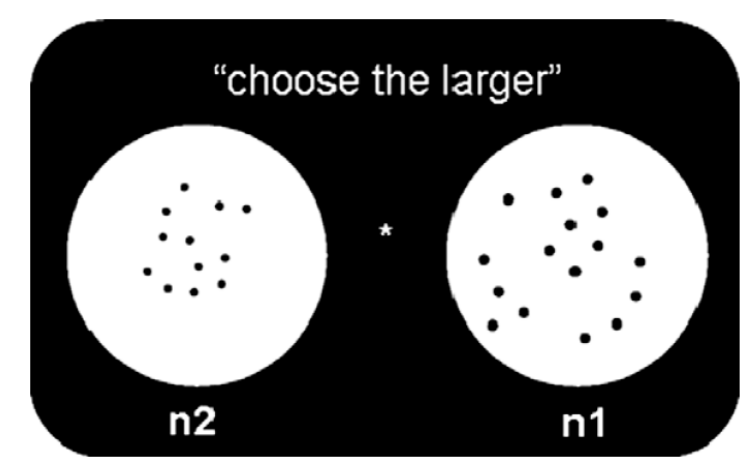

Figure 2. An example trial in non-symbolic comparison task (taken from Piazza et al, 2010). Two numerical sets are displayed. The participant has to indicate the numerically larger set without counting.

The ability to compare non-symbolic quantities, also known as number acuity, is usually assessed by calculating the Weber fraction $-w$ - (Halberda et al., 2012; Halberda, Mazzocco, \& Feigenson, 2008; Halberda, 2011; for other indexes see, Inglis \& Gilmore, 2014; Piazza et al., 2010). The calculation of the Weber fraction can vary according to the underlying model for the Approximate Number System. Nevertheless, the Weber fraction can be considered as the constant standard deviation ("noise") within a Gaussian curve; this corresponds to an internal representation of each numerosity on the mental number line (Halberda 2011). Therefore, the smaller the standard deviation (i.e., Weber fraction), the more precise the numerical representation. To put it concretely, an individual with a Weber fraction of .14 can reliably individuate the larger between two numerical sets, when one of the two numerosities is at least $14 \%$ larger than the other one. Then, for example, the individual can reliably discriminate 7 vs. 8 and 14 vs. $16\left(\mathrm{~N} 1=7,7^{*} .14=1, \mathrm{~N} 2=7+1=8\right.$; similarly, $\mathrm{N} 1=14,14 * .14=2, \mathrm{~N} 2=14+2=16)$.

As discussed earlier with regards to study of infant numerical cognition, a crucial issue is whether individuals actually extract numerical information from the presented visual stimuli (e.g., sets of dots) or base their discriminations on other non-numerical features that covariate with numerosity, such as total surface area or perimeter (Clearfield \& Mix 1999; Clearfield \& Mix 2001; Gebuis et al. 2014). For the most tightly controlled comparison task, 
the two presented numerical sets should be perfectly matched for all non-numerical variables and differ only in the number of items. This strict control would guarantee that individuals base their discrimination exclusively on the numerical dimension. Regrettably, the simultaneous control for all non-numerical variables is difficult to achieve. Researchers have tried to prevent participants form relying on non-numerical information by alternatively manipulating physical characteristics of the stimuli from trial-to-trial (Piazza et al. 2010), or creating numerical arrays that minimize the correlation between non-numerical variables and numerosity (Gebuis \& Reynvoet 2011; Gebuis \& Reynvoet 2012a). Indeed, work with adults has demonstrated that non-numerical characteristics of visual arrays biased numerical discrimination. However, numerosity was the crucial information that drove discrimination process (DeWind et al. 2015), thereby confirming the role of numerosity as primary visual property (Stoianov \& Zorzi 2012; Burr \& Ross 2008). Nevertheless, the debate on the influence of non-numerical features on non-symbolic numerical discrimination is still ongoing (Gebuis et al. 2014; Gebuis et al. 2016; Leibovich et al. 2016).

Symbolic number comparison: distance and size effect.

While the ability to discriminate between non-symbolic numerical quantities appears to be innate, young children have to learn how to correctly compare symbolic quantities (i.e., Arabic digits). With the acquisition of counting skills, children learn how to map nonsymbolic quantities to the corresponding symbolic representations. However, the mastering of the cardinality principle does not guarantee that children can also correctly individuate the larger between two digits (Le Corre \& Carey 2007; Le Corre 2014). As observed by Le Corre (2014), for successfully comparing number words between six and ten, cardinal-principle knowers must be able to map (i.e., verbally estimate) visually presented numerosities between six and ten to the counting list. Children need to create a reliable mapping between numerosities larger than 4 and the counting list to also understand the magnitude relation between digits. In the assessment of number comparison skills, two numbers (1-or 2-digit 
numbers) are usually presented respectively on the left and on the right side of the screen and participants have to choose the larger (or the smaller) by pressing the corresponding keyboard key. With expertise, the comparison of digits becomes a relatively simple task in which the vast majority of children, except for those with severe difficulties, reach an almost perfect performance. Therefore, RTs are usually assessed to obtain a meaningful measure of number comparison skills. Children between 6 and 8 years-old show a consistent decrease in RT as a function of age (Holloway \& Ansari 2009). More interestingly, both accuracy and RT change as a function of the numerical distance of the two presented numbers (i.e., Numerical Distance Effect, NDE; Moyer \& Landauer, 1967). RTs tend to increase when the numerical distance between the two displayed numbers decreases; therefore, the 2 vs. 3 (distance equals 1) comparison yields slower responses compared to 2 vs. 8 comparison (distance equals 6). The NDE is also observed in typically developing individuals who know which is the larger between the displayed numbers. Nevertheless, it takes more time to disentangle which is the larger digit when the two digits have small numerical distance. The NDE has been interpreted as a direct connection between the symbolic and non-symbolic representation of numbers.

The visual presentation of a symbolic number immediately activated the corresponding nonsymbolic numerical representation that is, accordingly to the Approximate Number System, a distribution of activation on a compressed number line (but see Zorzi \& Butterworth 1999; Butterworth 2010). Therefore, Gaussian curves of two close numbers (e.g., 4 and 5) are substantially overlapping, whereas the overlap of distributions of numbers that are far apart is minimal (e.g., 2 and 9). Besides the number comparison task, the NDE has been observed in other numerical tasks, such as numerical priming, naming and parity judgment task (Reynvoet et al. 2002; Brysbaert, W Fias, et al. 2002; Reynvoet \& Brysbaert 1999; Reynvoet \& Brysbaert 2004).

The comparison of large quantities is also slower compared to smaller numbers when the numerical distance is kept constant (i.e., numerical size effect, NSE). For example, 
individuals are faster in choosing the larger number in the comparison 2 vs. 3 than 8 vs. 9 despite the numerical distance being equal to 1 in both comparisons. The NSE is also considered a consequence of the Approximate Number System in which numerical representations tend to overlap more with the increase of numerical magnitude. However, some authors argued that the NSE is related to the requirements of the number comparison task rather than to an activation of the internal numerical representations. In fact, some studies have failed to find the NSE in naming and parity judgment tasks (Brysbaert, Wim Fias, et al. 2002; Reynvoet et al. 2002). Accordingly, Verguts, Fias, and Stevens (2005) proposed an alternative account to address the presence of NDE and NSE in the digit comparison task, and the absence of NSE in other numerical tasks. The NSE may be specifically related to digit comparison because the task requires mapping numbers onto the mental number line and to the task relevant output components. Thus, the NSE, in this model, does not stem from the representation level, but at a later response-related level.

In summary, the ability to enumerate and compare small non-symbolic numerical quantities is a fast and accurate process that relies on the Object Tracking System and in turn appears to be connected to VSTM capacity. The crucial signature of this system is its reduced capacity (up to 3-4 elements). The processing of larger numerical quantities appears to rely on the Approximate Number System, which entails "noisy" representations; therefore, the ratio between the presented numerical sets is the crucial factor for a successful discrimination. Interestingly, behavioural results from numerical estimation, numerical production and numerical re-production suggest that these processes also rely on the Approximate Number System. The comparison of symbolic quantities is characterized by the NDE and NSE, which are suggested to reflect the link between non-symbolic and symbolic numerical processing. 


\section{Numbers and space}

\section{The SNARC effect}

Experimental evidence has highlighted that numbers (and non-symbolic quantities, see below) are spatially represented, with small and large numbers respectively linked to the left and right side of the space. The Spatial Numerical Association Response Code (SNARC; Dehaene, Bossini, \& Giraux, 1993) is arguably the most widely investigated effect highlighting the intimate relation between numbers and space. As originally reported by Dehaene and colleagues (1993), participants provide faster responses with the left hand when judging relatively small numbers, and faster responses with the right hand when judging relatively large numbers. To put it concretely, when asked to judge whether a centrally presented digit is larger or smaller than a reference, for example 5, by pressing two different keys, participants are faster in responding with the left hand when small numbers $(<5)$ are presented, and faster in responding with the right hand when large $(>5)$ numbers are presented. The SNARC effect has been also observed when numerical information was completely irrelevant to accomplish the task. For example, the SNARC effect was found when participants are instructed to respond whether or not the presented number contains the “e” sound letter (Fias, Brysbaert, Geypens, \& D’Ydewalle, 1966), or they have to judge whether the presented number is odd or even (i.e., parity judgment task). Moreover, the SNARC effect emerges independently from the number format (auditory number words, visual Arabic numerals, visual number words, visual dice patterns; Nuerk, Wood, \& Willmes, 2005). It has been suggested that the SNARC originates from the spatial ordering of numbers, and the left to right reading direction in Western cultures further reinforce this disposition. In line with this hypothesis, Palestinians, who read both numbers and words from right to left, displayed a reversed SNARC effect when performing a parity judgment task (Shaki et al. 2009). Nonetheless, the SNARC effect appears to be extremely flexible and easy to reverse: 
for example, Russian-Hebrew bilinguals showed a reversed SNARC effect immediately after reading a Hebrew text (from right to left; Shaki \& Fischer, 2008).

The emergence of the SNARC effect only after some years of school suggests that number-space association can be the consequence of cultural influence, such as reading direction (Berch, Foley, Hill, \& Ryan, 1999; for similar results, van Galen \& Reitsma, 2008). Nevertheless, preschool children seem to display a left to right number-space association even before formal math education and the acquisition of reading skills (Hoffmann et al. 2013; Opfer \& Thompson 2006). The number space interaction has been found also for nonsymbolic numerical quantities in young children. Accordingly, preschool children showed faster reaction times to smaller sets presented on the left side of the screen and to larger sets presented on the right side (Patro \& Haman, 2012; for number-space association in infants see, de Hevia \& Spelke, 2010). Other behavioural tasks have highlighted an intrinsic relation between numbers and space. For instance, Fischer and colleagues (Fischer et al. 2003) reported that centrally presented digits can orient participants' attention and facilitate the detection of lateralized target stimuli. Crucially, small numbers oriented participants' attention to the left side of the space whereas larger numbers $(>5)$ oriented the attention on the right side of the space.

Insights from the relation between number and space come also from the neuropsychological studies of patients with neglect. In this clinical condition, patients are affected by a brain damage (usually) on the right, posterior side of the brain, which leads to a failure in directing attention to the contralesional (left) hemi field. Following this evidence, Zorzi and colleagues (Zorzi et al. 2002) investigated spatial representation of numbers in patients with neglect by asking them to solve a verbal numerical bisection task. Patients were verbally presented with two numbers that corresponded respectively to the lowest and highest extremes of a numerical interval (e.g., 1 and 9). Patients had to indicate the number in the middle of the interval without using any calculation or counting strategy. Interestingly, 
patients with neglect constantly overestimated the position of the middle number in the numerical interval (e.g., 7 in the interval 1-9). This result suggested that patients failed to focus their attention on the left part of the numerical interval, and provided their estimations solely basing on the rightmost part of the interval, thereby showing the consequent overestimation. In the physical bisection line task, healthy participants see a small or larger numbers aligned to compose a straight horizontal line (e.g., 2222222222222) and are asked to mark the middle point of the line. Participants show a biased bisection toward left when the line is composed of small digits, whereas the bisection is biased toward right in the case of large digits (Fischer 2001).

At least three different theoretical accounts have been proposed to interpret the behavioural findings related to the number space association. According to the Mental Number Line (MNL) account, numbers are represented on a putative horizontal line whose orientation (left-to-right or right-to-left) is heavily influenced by cultural features and experience. The encoding of an Arabic digit automatically elicits the spatial location of that specific number and moving along the MNL involves spatial attention (cf, Doricchi, Guariglia, Gasparini, \& Tomaiuolo, 2005; Fischer et al., 2003). Nevertheless, the MNL account is insufficient to fully explain the flexibility of the SNARC effect. Another theoretical account interprets the number-space interaction as a consequence of the verbal association between a stimulus property (i.e., small or large) and the response category (i.e., left or right). Numbers are firstly coded in to the verbal dimension large-small and then associated with the left-right spatial dimension in a conceptual-polarity coding (Proctor \& Cho 2006; Santens \& Gevers 2008; Gevers et al. 2010). In contrast with the MNL account, polarity coding well explains the observed flexibility of the SNARC effect, but lacks a solid explanation for the other behavioural results such as those from the bisection tasks. Finally, the working memory (WM) account states that ordinal information, such as numbers (but also other ordered sequences), are spatially stored in working memory (van Dijck et al. 2009; Fias 
et al. 2011). The association between numbers and space is created online when individuals perform the proposed tasks, thereby explaining the flexibility of the SNARC effect. Taken together, the explanations provided by the aforementioned three accounts fail to fully describe all the behavioural and neuropsychological results reported by the previous literature. It appears that the relation between numbers and space entails a complex scenario in which different mechanisms and cognitive processes (verbal WM, visuo-spatial WM, spatial attention) play a crucial role (for a comprehensive model see, van Dijck, Gevers, Lafosse, \& Fias, 2012).

\section{Number Line mapping}

Another way to link numbers to space is by representing numbers as a spatial position within a visual horizontal line. In the Cartesian axes system, numbers are arranged on a line in which the spatial information conveys numerical magnitude. Therefore, the distance between 2 and 3 equals the distance between 8 and 9, thereby respecting a linear relation between numerical quantities. In a seminal study, Siegler and Opfer (2003) asked children and adults to mark the position of several target numbers onto two horizontal lines (also known as Number Line task, Figure 3) entailing the interval from 0 to 100 and from 0 to 1000. In the 0-100 interval, children from the second grade displayed a fairly accurate and linear mapping whereas, in the 0-1000 interval, they overestimated the position of small numbers and slightly underestimated the position of larger numbers, thereby yielding a biased (log-like) pattern of estimates. Older children and adults, instead, mapped numbers near the correct locations, thus, showing a linear and accurate positioning (Berteletti et al. 2010; Siegler \& Booth 2004; Siegler \& Opfer 2003). Similar results were observed by Berteletti and colleagues (2010) who presented three numerical intervals (i.e., 1-10, 1-20, 0-100) to pupils from 4 to 6 years of age. Again, young children displayed a linear mapping in the interval 1-10, whereas a biased (log-like) mapping was observed for larger intervals. Individual mapping analysis highlighted that children shifted from a biased (log-like) to an 
accurate (linear) mapping with age. The same shift from biased to linear mapping has been observed for discrete numerical quantities and for other ordered sequences (Sella et al. 2015b; Berteletti et al. 2012). The shift from a biased to an accurate mapping of numbers has been originally explained as the consequence of a shift from a logarithmic to linear representation of numbers (Siegler \& Opfer 2003). Other theoretical accounts have linked the shift to an increasing ability in performing proportional judgements (Barth \& Paladino 2011; Slusser et al. 2013) or the increased knowledge and familiarity with both the presented numbers and numerical intervals (Ebersbach et al. 2008; Hurst et al. 2014; Moeller et al. 2009).

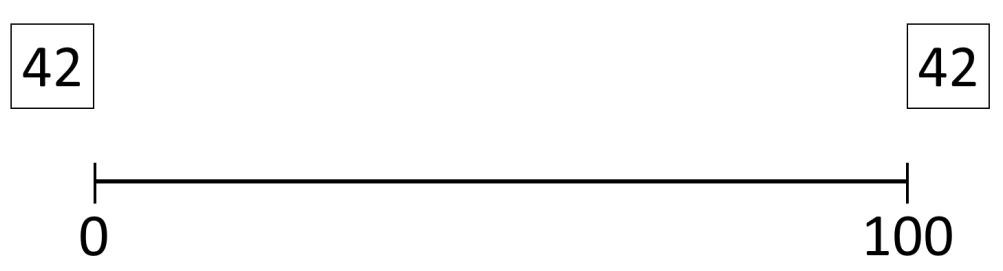

Figure 3. An example of a trial in the Number Line task: Participants have to mark on the line the position of the target number (in the upper left and right corner).

In sum, several behavioural results have highlighted the intrinsic relation between space and number. Regarding the SNARC effect, while the MNL account emphasises the long-term acquired left-to-right spatial representation of numbers, other accounts, such as the Polarity-coding and the WM, have suggested that the number-space association is created on line and characterized many other sequences (e.g., days of the week). Nevertheless, the complexity of the relation between numbers and space may need a wider and more extended theoretical framework. Another way to connect numbers and space is represented by the ability to accurately map numbers onto a visual line, as in the number line task. Young children usually display a log-like biased mapping of numbers that tends to linearize with age and expertise. 


\section{The interaction between numerical and physical magnitude: the Size Congruity effect}

In everyday life, numerical and other physical quantities tend to be correlated. Large numerical sets occupy more space (both perimeter and surface area) compared to smaller ones. For this reason, the vast majority of studies involving the discrimination of numerical quantities tried to control for the effect of physical dimensions. Does the relation between magnitude and numerical quantities also hold for Arabic numbers? Do symbolic and physical representations of magnitudes interact? To investigate this issue, authors have implemented a numerical-like Stroop task in which numerical and physical information are varied independently (Tzelgov et al. 1992; Algom et al. 1996; Cohen Kadosh \& Henik 2006; Henik \& Tzelgov 1982). In such a task, individuals have to choose the physically larger of two presented digits while ignoring their numerical values. Responses are faster when the physically larger digit is also numerically larger (i.e., 8 vs. 2 , congruent trial) compared to the opposite condition in which the larger digit has the smaller physical size (i.e., 2 vs. 8 , incongruent trial). This Size Congruity Effect (SiCE) is also observed when individuals have to choose the numerically larger digit while ignoring the physical size. From this, it can be concluded that the processing of both numerical and physical quantities is automatic and individuals cannot ignore the influence of one on the other. Girelli, Lucangeli, and Butterworth (2000) found that the SiCE gradually emerges after the third year of primary school and becomes more marked with age. Rubinsten, Henik, Berger, and Shahar-Shalev (2002) have found that the SiCE appears at the end of the first year in primary school. This indicates that children need numerical knowledge and experience to achieve an automatic processing of Arabic digits, which, in the pre-counting phase, are arbitrary symbols lacking any specific numerical meaning (Gebuis et al. 2009).

Two alterative theoretical explanations have been proposed for explaining the SiCE (Cohen Kadosh, Cohen Kadosh, Linden, et al. 2007; Schwarz \& Heinze 1998). The shared 
representation account claims that physical and numerical dimensions are jointly represented in the same analogical system. Therefore, when the numerical and the physical dimensions diverge (i.e., incongruent trial), the same system takes more time to disentangle contrasting information and to create a shared magnitude of physical and numerical information. This account fits the idea that different magnitudes, such as time, space and numbers, share a common representation (Walsh 2003; Bueti \& Walsh 2009). The shared decision account, instead, claims that the processing of numerical and physical information is mainly parallel and only reaches an interaction at the response selection level. Therefore, in the incongruent trials the numerical and physical magnitudes activate two separate magnitudes and contrasting response codes which interfere (i.e., longer RTs) in the decision phase. Current evidence supports the existence of both accounts, which might be related to task-difficulty (Cohen Kadosh, Cohen Kadosh, Linden, et al. 2007).

\section{Arithmetic facts and mental calculation}

Children use counting to establish a solid link between symbolic and non-symbolic representations of numerical quantities. This connection is extremely important because it allows children to acquire the cardinal meaning of symbolic numbers (Wynn 1990). However, only few months after understanding the cardinal meaning of numbers, children also learn that the later number-word in the numerical sequence corresponds to a larger numerical quantity (Le Corre 2014). Thereafter, children can operate on Arabic numbers based on magnitude meaning to develop a series of basic symbolic numerical skills. For example, children at this stage of development can determine (with different levels of accuracy and speed of response) the larger between two visually or orally presented Arabic digits, or can order numbers from the smallest to the largest or vice versa. Subsequently, children still draw upon their counting skills in combination with fingers to solve simple arithmetic problems, such as one-digit additions and subtractions (Crollen \& Noël 2015; 
Moeller et al. 2011; Thompson 1999). For young children, all basic calculations require a strong involvement of control processes as well as procedural and numerical knowledge. For solving " $5+4$ ", children need to know the meaning of the numerical sign " + " and the associated procedure (i.e., counting forward). Counting at this stage is a cognitively demanding process that is usually supported by fingers or verbal reciting. After a period of intense rote learning during schooling, the solutions of elementary arithmetic problems are progressively stored in (verbal) long-term memory along with multiplication tables (Dehaene \& Cohen 1997; Hittmair-Delazer et al. 1994; Whalen et al. 2002; Ashcraft 1982), thereby constituting a repertoire of easy-to-retrieve arithmetic facts. The shift between a countingbased to a retrieval-based strategy in basic arithmetic problem solving usually occurs between the second and third grade of primary school (Geary et al. 1987; Ashcraft \& Fierman 1982). The distinction between arithmetic facts and calculation procedures is also confirmed by clinical studies in which neurological patients demonstrated a complete loss the of the procedures related to arithmetic, but displayed preserved knowledge of arithmetic facts (Delazer \& Benke 1997). When trained in learning new arithmetic facts, adults display an increased activation in brain areas related to effort and control (i.e., frontal areas) for untrained arithmetic problems and an increased activation of memory related areas (i.e., angular gyrus) for trained arithmetic problems (Ischebeck et al. 2009; Delazer et al. 2005; Grabner et al. 2009). Arithmetic facts are considered to be important as calculations become more complex. When asked to solve a complex arithmetic problem (e.g., 24 x 37), in order to provide the correct solution, individuals have to encode the problem characteristics (e.g., arithmetic symbols), to apply the best computational strategy (e.g., adding decades and units separately), to retrieve arithmetic facts while partial results are kept active in memory. The processes (Caviola et al. 2012; Grabner et al. 2009; Lemaire 1996; Logie et al. 1994; McLean \& Hitch 1999; Passolunghi \& Cornoldi 2008). Therefore, automatized retrieval of arithmetic facts can release resources that can, in turn, be allocated to other cognitive processes, which 
are necessary for accomplishing complex calculations. When the solution of an arithmetic problem (e.g., $37+49)$ is not stored in the long term memory, individuals have to draw upon mental or written calculation. Mental calculation heavily relies on the use of strategies to transform the presented problems in subcomponents that are easier to manage. For instance, decomposing " $37+49 "$ in " $30+40=70 ", " 7+9=16$ ", and finally " $70+16=86$ ". Several taxonomies of strategies and the frequency of their implementation have been investigated (Lucangeli et al. 2003; Threlfall 2000; Thompson 2000). The implementation of mental calculation strategies is intimately linked to executive and working memory capacities, especially the visuospatial component (Passolunghi \& Siegel 2001; DeStefano \& LeFevre 2004; Mammarella et al. 2010; Imbo et al. 2007; Sella et al. 2012).

In summary, school-aged children initially rely on counting strategies to solve simple single-digit arithmetical problems. The repeated association between arithmetic problems and their answers leads children to progressively store arithmetic problems (e.g., multiplication table) along with the correct answers in verbal long-term memory, thereby developing a repertoire of arithmetic facts. The accurate and efficient retrieval of arithmetic facts is a key component when children and adults have to solve more complex mental calculations. Moreover, an efficient retrieval of arithmetic facts can leave free resources that can potentially be allocated to the selection and execution of efficient computational strategies. The acquisition of arithmetic facts and achievement of complex mental calculation heavily rely on the memory system in its verbal, visuospatial, and executive components.

\section{Numerical processes and mathematical achievement}

Stemming from the evidence of innate numerical skills (Antell \& Keating 1983; Izard et al. 2009; Xu \& Spelke 2000), some authors have investigated the contribution of nonsymbolic numerical processing on mathematical competences (Dehaene 2011). In this vein, the basic representations of numerical quantities constitute the foundation upon which 
children build advanced numerical and mathematical skills. The ability to rapidly enumerate small numerical quantities, namely subitizing, has been correlated with mathematical achievement. Accordingly, children with developmental dyscalculia (a clinical condition characterized with poor mathematical achievement) display a lower subitizing limit compared to typically developing peers (Landerl 2013; Schleifer \& Landerl 2011). Children with Down syndrome, who usually display low math achievement, have poor subitizing capacity compared to children matched for both mental and chronological age (Sella et al. 2013). In young children, subitizing, along with executive skills, has been found to contribute to the developing of counting and enumeration skills (Kroesbergen et al. 2009), which in turn constitute a fundamental predictor of later math achievement (Reeve et al. 2012). The ability to compare large numerical quantities - number acuity - has been repeatedly associated with math achievement. Halberda, Mazzocco, and Feigenson (2008) found that number acuity remained a significant predictor of performance in standardized mathematical achievement tests also when the effect of several other cognitive processes was statistically controlled. Accordingly, children with math learning disability exhibit a reduced performance in comparing non-symbolic quantities (Piazza et al. 2010; Mazzocco et al. 2011a). Moreover, number acuity has been found to be a significant predictor of later mathematical achievement (Mazzocco et al. 2011b; Feigenson et al. 2013; Starr et al. 2013; Libertus et al. 2013). Therefore, an accurate representation of non-symbolic numerical quantities constitutes an important predictor of later mathematical achievement. A "noisy" representation of nonsymbolic numerical quantities can compromise the acquisition of subsequent numerical skills and math achievement. Nevertheless, some authors have claimed that the link between number acuity and math achievement can be explained as a consequence of poor inhibition of those physical variables that covary with numerosity (Clayton \& Gilmore 2014). Gilmore and colleagues (2013) found a significant correlation between accuracy in comparing sets of dots and mathematical achievement. Importantly, this was only for those trials in which the more 
numerous array was composed of smaller dots and occupied a smaller area (i.e., incongruent trials). A lack of correlation was observed for those trials in which the more numerous array comprised larger dots and had a larger occupied area (i.e., congruent trials). Moreover, the correlation between math achievement and accuracy in incongruent trials disappeared when a measure of inhibition was taken into account. Therefore, the ability to inhibit the influence of non-numerical features of the presented numerical sets, rather than the acuity of the Approximate Number System, appears to be related to math performance (Figure 4).

\section{$<$ FIGURE 4 ABOUT HERE $>$}

<FIGURE 4 FROM: Gilmore et al., 2013>

Other studies have highlighted the role that access to numerical magnitude through symbolic numbers can influence the development of numerical and mathematical skills (Butterworth 2010; Reeve et al. 2012; Bugden \& Ansari 2011). Rousselle and Noel (2007) found that children with math learning disability demonstrated poorer performance (i.e., slower RT and accuracy) when comparing Arabic digits, as well as marginally reduced size and distance effect, compared to a group of typically developing children. Conversely, no difference was observed in terms of non-symbolic numerical comparison (i.e. dots comparison). Two other studies have highlighted that children with math difficulties showed a deficit in symbolic number comparison (by means of an increased NDE), whereas no differences emerged for non-symbolic processing (Holloway \& Ansari 2009; De Smedt \& Gilmore 2011). However, Mussolin, Mejias and Noël (2010) observed that children with developmental dyscalculia displayed a larger NDE compared to typically developing children, irrespective of the number format (i.e., Arabic numerals, number words, canonical and non-canonical dots patterns. In a large sample study on primary school children, Lyons and colleagues (2014) found that the ability to judge the ordinality (from left to right) of 
Arabic numbers mainly contributes to mathematical achievement rather than non-symbolic numerical skills (for a review see, De Smedt, Noël, Gilmore, \& Ansari, 2013).

The size congruity effect emerges with increasing numerical expertise (which is confounded with development and changes in cognitive control); however, it lacks a consistent correlation with math achievement (Bugden \& Ansari 2011; Gebuis et al. 2009; Sella et al. 2016). Similarly, a systematic review of the existing literature has shown a lack of any consistent association between the SNARC effect and mathematical achievement (Cipora $\&$ Nuerk 2013). A recent study showed that mathematicians did not display a SNARC effect, thereby suggesting the presence of an extremely flexible spatial representation of numbers (Cipora et al. 2015). Conversely, the ability to accurately map numbers on the line was repeatedly associated with mathematical achievement (Booth \& Siegler 2006; Booth \& Siegler 2008; Sasanguie et al. 2013; Geary et al. 2009). Accordingly, children with math disability display a less accurate mapping in the NL task compared to typically developing children (Geary et al. 2008; Landerl et al. 2004; Sella et al. 2014). Also adult mathematicians displayed a more accurate performance in the NL task compared to non-mathematicians (Sella et al. 2016). Training programs have been developed to improve the ability to spatially map numbers. Simple linear board games can enhance spatial mapping as well as digit comparison, counting and number identification in preschool children from low socioeconomic status (Ramani et al. 2012; Siegler \& Ramani 2008; Siegler \& Ramani 2009). Number line trainings have provided good evidence in improving numerical skills also in children with math learning disability (Kucian et al. 2011). Moreover, computerized software has been developed using the spatial number line structure as a key component, which has already demonstrated to be a valid tool for improving numerical skills in children of different ages (for a review see, Moeller, Fischer, Nuerk, \& Cress, 2015).

Mathematical achievement is not only based on basic numerical representations but requires other components such as arithmetic procedural knowledge, knowledge of arithmetic 
facts, computational strategies and problem solving skills. Mathematical achievement is the heterogeneous product emerging from the combination of specific numerical skills and different domain processes (Passolunghi et al. 2014; Soltész et al. 2010; Szücs et al. 2013; Szücs et al. 2014). In this vein, Szücs and colleagues (2014) assessed mathematical achievement along with domain-specific and domain-general cognitive processes in a large sample of 9 years old children. What emerged was a crucial role for of an "executive memory function centric model" in the acquisition of mathematical competences, whereas the impact of basic numerical processes was limited. General intelligence is widely accepted as a precursor of mathematical competences and, more broadly, of learning and scholastic success (Deary et al. 2007; Spinath et al. 2006). In a longitudinal study, Geary (2011) observed that intelligence, speed of processing and the executive component of working memory predicts achievement both in mathematics and reading. The visuospatial component of working memory specifically predicted mathematical competences, while early arithmetic skills were important for later mathematics achievement, above and beyond the influence of domaingeneral components. Finally, language related abilities, such as phonological awareness and reading skills, have been found to contribute to the development of mathematical competences (Koponen et al. 2007; Lee et al. 2004).

In summary, mathematical achievement can be conceived as a heterogeneous product resulting from the combination of specific numerical processes and general cognitive skills. Research has shown that both domain-general and domain-specific cognitive processes contribute to mathematical learning (Fuchs et al., 2010; Passolunghi \& Lanfranchi, 2012; Träff, 2013). 


\section{InSIGHTS FROM NeUROPSYCHOLOGY \& COGNITIVE NeUROSCIENCE}

\section{A Brief Overview of Neuroscientific Approaches to Studying Numerical Cognition}

As with Experimental Psychology more broadly, early understanding of the human brain was derived from neuropsychological evidence, where the damaged brain was used to link behaviour to the underlying neural anatomy. Without use of the powerful neuroimaging tools that are available today, however, early approaches comprised cataloguing deficits that were localised to damage based on external signs of injury, surgical information, or lesions identified post-mortem. Modern neuropsychology has benefited from significant scientific advances enabling imaging of the brain in vivo, wherein a lesion or damaged connection can be precisely mapped using high-resolution structural or diffusion-weighted images - indices from which can be related to behaviour - providing a dynamic view of brain and function. One approach here is to examine the behaviour of individuals who have in common the anatomical location of a lesion which, for numerical cognition, would typically be in the parietal, temporal and occipital lobes. Another approach is to seek patients who feature the same constellation of behavioural symptoms or syndrome, then identify collective areas of brain damage or disconnection, for example, through voxel-based lesion-symptom mapping (see Bates et al. 2003 for an overview). Such work attempts to operationalise numerical cognition by examining deficits that are specific to numbers, such as acquired acalculia or anarithmetia, and seeking to dissociate numerical processes from interdependent systems for language and visuo-spatial reasoning, for example. Neuropsychological study has informed various models of numerical processing, including the influential TCM (Dehaene \& Cohen 1995; Dehaene \& Cohen 1997), which we later summarise.

Similarly to patient studies, non-invasive brain stimulation (NIBS) is used to make causal associations between brain structure and function. There are a number of forms of NIBS, which will be discussed in this chapter, and these are broadly categorised as transcranial magnetic stimulation (TMS) and transcranial electric stimulation (tES) (for an 
overview of mechanisms, see Vallence \& Ridding 2014). Such techniques are used to produce a transient change in behaviour, which, depending on the protocol, could be a reduction or enhancement in function. This change results from the applied, temporary interference (or facilitation) that NIBS induces in the targeted brain region or network. The primary advantage of NIBS over classic lesion studies is that a more precise region, or regions, of the brain can be studied in healthy adults, avoiding the complex task of determining anatomical similarity across what are heterogeneous areas of damage, which is more typical when working with patients. Moreover, participants can be selected on the basis of demographic or behavioural similarity. By seeking individuals who share socioeconomic, ethnic or cognitive features, it is possible to minimise non-experimentally induced differences in how each individual responds to stimulation (for a review, see Krause \& Cohen Kadosh 2013). In addition, NIBS produce transient change in brain functions, and therefore can reveal the casual link between brain and behaviour without any compensation that might occur in the case of the damaged brain.

NIBS within the field of numerical cognition has typically been centred on the parietal, dorsolateral and prefrontal cortices. NIBS has been used to examine basic numerical processes, for example, to dissociate numerical systems or processes (e.g., Cappelletti et al. 2007; Cohen Kadosh et al. 2010; Javadi et al. 2014), to reproduce the behavioural characteristics of numerical disability (Cohen Kadosh, Cohen Kadosh, Schuhmann, et al. 2007) or math anxiety (Sarkar et al. 2014), and to facilitate improvement in arithmetic tasks in typical (e.g., Hauser et al. 2013; Snowball et al. 2013; Popescu et al. 2016) and atypical (Iuculano \& Cohen Kadosh 2013) populations (for a comprehensive review, see Sarkar \& Cohen Kadosh 2016).

The pursuit of a cognitive neuroscientific account of numerical cognition has been aided considerably by functional brain mapping techniques. These primarily comprise, electrophysiological measurement obtained using electro- and magneto-encephalography 
(EEG, MEG respectively) and measures of experimentally induced metabolic change with Positron Emission Tomography (PET), or hemodynamic change, achieved using functional magnetic resonance imaging (fMRI) and functional near infrared spectroscopy (fNIRS) (for an overview of fNIRS, see Ferrari \& Quaresima 2012). As is widely covered in most introductory cognitive neuroscience textbooks, each technique has its own advantages and limitations: most notably, these reflect the trade-off that has to be made between superior temporal resolution, as is achieved with EEG/MEG, and anatomical specificity, as is attained with fMRI and fNIRS. Although lesser used, fNIRS provides some distinct advantages over fMRI. Principally, fNIRS has lower equipment costs and, being a cap-derived system, facilitates use with wider populations and experimental protocols that are inappropriate for use within a conventional MRI scanner. Regardless, quantitative comparison between the two techniques suggests that $\mathrm{fMRI}$ retains overall superior signal-to-noise ratio, which is reflected in improved spatial resolution (Cui et al. 2011). Functional brain mapping of numerical cognition has been used to highlight differences in cognitive processing that are not apparent at the behavioural level, for example, to illustrate systematic differences in passive visual processing of numerical versus non-numerical information (e.g., Eger et al. 2013), or where an overt behavioural response is unobtainable, such as is the case with pre-verbal infants (e.g., Hyde \& Spelke 2011). Similarly, functional brain mapping has also been informative to characterising divergent behavioural responses, such as the SNARC effect (Cutini, Scarpa, et al. 2014).

Indeed, the measures briefly described above can, and have been, combined to provide powerful, testable hypotheses regarding the neurocognitive bases of numerical cognition. The remainder of this chapter brings together evidence from neuropsychology and cognitive neuroscience to provide a brief overview of current understanding of how the brain processes numbers, looking first at evidence from early lesion studies, including an overview of the influential TCM. Next we discuss some of the core debates surrounding the 
representation of non-symbolic and symbolic number. Lastly, we talk about estimation and precise enumeration of quantity, with regard to the Approximate Number System and Object Tracking System. We concentrate on numerical cognition, however, we do draw on the wider mathematical cognition literature where appropriate.

\section{Exploring Numerical Cognition through Cognitive Neuropsychology}

This chapter is primarily focused on numerical cognition. Nonetheless, neuropsychological studies of acalculia, an acquired deficit in calculation, have helped to describe the brain networks that support numerical processes. Acalculia can result from a variety of concomitant syndromes including aphasia, alexia and agraphia, where calculation may be affected by damage to underlying language or other cognitive systems, rather than resultant from atypical numerical systems per se. Consequently, not all deficits with calculation reflect primary acalculia. Nevertheless, disentangling primary from secondary acalculia is informative to delineating the functional systems that support numerical processing.

A core feature of neuropsychological case studies of numerical cognition is that of impairment within a specific category of number. For example, Cipolotti et al. (1995) describe an acalculic patient, BAL, who, following several strokes in the left parietal lobe, was unable to read aloud Arabic numerals (e.g., "8"), yet unimpaired when reading aloud the corresponding written number word (e.g., "eight") and other non-number words. This deficit was not specific to any digit, but instead appeared to reflect the modality of visual presentation. Importantly, this impairment was not phonological, as the patient could formulate the required sound if presented the alphabetic number word. Thus, BAL was impaired in his ability to interpret the semantic meaning of symbolic (i.e. Arabic) number, which could otherwise normally be deduced from the alphabetic word form. A dissociation has also been demonstrated between the production of spoken versus written number. Marangolo et al. (2004) detail the case of a former accountant, patient NM, who was able to 
read aloud words from written categories including fruits, animals and furniture, yet impaired in reading aloud Arabic numerals and alphabetic number words. NM suffered no difficulty with producing written forms of number, however. Thus, unlike the previous case, NM demonstrated a complete inability to retrieve the phonological lexical representation of number, indicating that there are different processes supporting numbers and words for lexical retrieval. Other research has identified a patient presenting with the reverse pattern, wherein they could read aloud number words without error, but demonstrated difficulty with oral production of non-number words (Bencini et al. 2011). Together, these data suggest that the there is a specific lexicon for numbers and that this is "organized in a modality-specific way, with independent lexicons for orthography and phonology” (Marangolo et al. 2004). Cases of selective agraphia - an impairment in writing - provide a case in point that separate systems sub serve the production of numerical content in written versus spoken form. For example, Delazer et al. (Delazer et al. 2002) report the case of patient JS who, following a stroke in the left parieto-occipital cortex, suffered severe agraphia. Whilst JS was impaired in writing upper- and lower-case letters to dictation, he was errorless when writing Arabic digits. He demonstrated no difficulty with oral spelling, thus excluding the possibility that his agraphia might be due to a central deficit in generating the appropriate graphemes. This case was supported by Butterworth et al. (2001) who reported a the case of a patient presenting with the opposite pattern following left temporal lobe atrophy.

The demonstration of selective impairment in the representation of number more broadly suggests that number forms a category specific semantic domain that is dissociated from other classes of knowledge (Cipolotti et al. 1991; Thioux et al. 1998; Butterworth et al. 2001) and, as such, it would be reasonable to expect these dissociations to be supported by different, albeit possibly interacting, neural systems. Voxel-based lesion-symptom mapping has been used to demonstrate a distinction in functional localization of reading aloud number versus non-number words, where impairment in Arabic and alphabetic number word reading 
was primarily localised to temporo-parietal regions, and non-number word reading to more frontal regions, including Broca's area and the premotor cortex (Piras \& Marangolo 2009). This suggests that there are different neural systems required for the output of number versus non-number, and when reading Arabic and alphabetic numbers versus non-number alphabetic words.

What can be gleaned from the summarised work is that numerical information in its 'simplest' form - a single Arabic digit or number word, for example - is not equivalent in all modes of presentation. Furthermore, Arabic numbers are not supported by the same cognitive systems that represent alphabetised language. Numerical information is compartmentalised, and distinct domains exist at the neurocognitive level. Further distinctions have been identified in mathematical cognition. Warrington (1982) provided the first systematic, single case study of primary acalculia. Patient DRC sustained an intracerebral haematoma in the left posterior parieto-occipital cortex, which resulted in difficulty with simple arithmetic. Assessment of DRC's understanding of arithmetic operations demonstrated that he could accurately articulate arithmetic strategies - how to perform addition, subtraction and multiplication - but long response latencies and inconsistent responses for simple addition and subtraction suggested he had lost the automaticity of responses that might be expected to be retrieved from memory. In conclusion, Warrington (1982) states that DRC's impairment reflected "knowledge of arithmetical facts, not his knowledge of arithmetical operations" (p.45). This seminal study provided an early indication of the existence of multiple, distinct domains of knowledge at the arithmetic level, wherein conceptual knowledge of the procedural steps required to compute arithmetic tasks - which were intact in patient DRC could be distinguished from factual knowledge which would support immediate retrieval of arithmetic facts - for example, that $5+7=12$ - on which DRC was impaired. Indeed, later neuropsychological studies evidence that arithmetic knowledge is supported by functionally distinct capacities, which can be characterised according to procedural, conceptual and 
factual knowledge. This has been demonstrated by research showing the existence of deficits in arithmetic fact retrieval in a patient with spared procedural knowledge (McCloskey et al. 1985), the opposite pattern in a further patient (Rosca 2009), and an instance of impaired conceptual knowledge, yet preserved knowledge for numerical facts (Dehaene \& Cohen 1997).

\section{A System for Numerical Cognition \& Arithmetic Processing}

\section{Triple Code Model}

Formulated on the basis of adult neuropsychological evidence, and arguably the most influential account of numerical cognition, the TCM ( Dehaene \& Cohen 1995; Dehaene \& Cohen 1997) has stimulated much work towards a neurocognitive model of numerical and arithmetic cognition. Unlike other core models of numerical cognition, the TCM outlines several testable predictions, and has been subject recently to substantial review in functional imaging (Arsalidou \& Taylor 2011; Moeller, Willmes - von Hinckeldey, et al. 2015).

Therefore, the following section will summarise this particular model and some of the core evidence surrounding it.

The model assumes three distinct, but interconnected, categories in which mental representations of numbers can be processed: i) a visual Arabic code, where numbers comprise strings of digits; ii) a verbal word code, where the syntactic organisation of numbers are represented as ordered sequences of words; iii) an analogical magnitude code, for the understanding of quantity and magnitude that is associated with a number. On the basis of neuropsychological evidence, the model outlines the anatomical bases for each of these three categories, wherein the visual number system is represented in an occipitotemporal network, corresponding to the ventral visual pathway. Whilst this system is outlined bilaterally, the authors suggest that visual number representation is primarily computed by the left hemisphere, co-opting neural systems which support object recognition. The verbal 
word frame is left lateralised, following wider population norms in language lateralisation. In order to produce or identify a sequence of spoken numbers, classic language areas are recruited including the perisylvian network - inferior frontal, superior and middle temporal gyri, plus a subcortical circuit comprising the basal ganglia and thalamic nuclei. A bilateral parieto-temporo-occipito network is suggested to facilitate representations of magnitude and comparisons of quantity, including estimation and subitizing.

Dehaene and Cohen (1997) propose that simple arithmetic problems can be solved using one of two routes. The first is a direct asemantic route to rote memory such that $2 \times 4$, for example, would trigger the verbal representation and any associated completion, "two times four, eight”. This route is suggested to involve a left cortico-subcortical loop responsible for storing sequences of rote learned arithmetic facts, and is thus likely to reflect simple single digit sequences for multiplication and addition. The second route is an indirect semantic route but, unlike the first, can represent values on which semantically meaningful operands can be executed, therefore providing a route when rote memory is unavailable. This is postulated to reflect the process supporting complex addition, division and subtraction, wherein overlearned verbal sequences are unlikely to exist. In this instance, the operands are encoded as quantity representations stored in the inferior parietal areas. Arithmetic manipulations can then be performed on these internal quantity representations, returning a result from the left inferior parietal cortex and perisylvian language network for naming. For complex tasks, numerical representation may be supplemented with processes that are not specific to number by the prefrontal cortex. Note that, within this framework, the simultaneous operation of both routes is stated as possible. In sum, the model predicts that two major processes responsible for calculation: a direct, asemantic route that is underpinned by a cortico-subcortical loop from which simple arithmetic facts can be retrieved from rote verbal sequences and, for more complex processing where direct memory retrieval is not possible, an indirect semantic route that additionally includes posterior parietal regions 
supporting number manipulation and the bilateral inferior parietal cortices which support semantic knowledge about numerical quantity.

There is some evidential consistency with the anatomical correlates and functions described by Dehaene and Cohen $(1995,1997)$, where the parietal lobe - particularly the intraparietal sulcus (IPS) - has been broadly implicated in processing quantity and magnitude comparison (see reviews by Cohen Kadosh et al. 2008; Cantlon et al. 2009). Over time, however, as interest in numerical cognition has grown, and with the increased accessibility of advanced neuroimaging techniques, the original network described in the TCM has been subject to modification. Using Activation Likelihood Estimation, Arsalidou and Taylor (2011) sought to re-evaluate the grey matter correlates of number and calculation tasks on the premise of comparing their meta-analytic findings with the TCM. The authors report that mathematical performance is associated with brain areas that have been extensively studied under the model, but that there are other areas - particularly the cingulate gyri, insula and cerebellum - that are regularly identified in number and calculation tasks (see figure 5). Similarly, the general premise of numerical cognition being sub served by a frontoparietal network was also supported in a recent review of structural and functional connectivity, implicating fronto-parietal association fibres generally, plus commissural fibres to connect the bilateral IPS when numerical magnitude information was processed (Moeller, Willmes - von Hinckeldey, et al. 2015).

\section{$<$ FIGURE 5 ABOUT HERE $>$}

$<$ FIGURE 5A FROM: Arsalidou and Taylor (2011); 5B from Moeller, Willmes - von Hinckeldey, and Klein (2015)>

The distinction between processes for rote learned versus procedural arithmetic tasks has also found support, where functional neuroimaging indicates that single-digit 
multiplication recruits language areas, whereas subtraction recruits the IPS (Zhou et al. 2007; Prado et al. 2011; Andres et al. 2012). It is important to point out, however, that the evidence base for a distinction between the direct asemantic route and indirect semantic route does not equal evidence for a neural distinction between mathematical operands (i.e. + versus $\mathrm{x}$ ) or surface complexity - the perceived level of difficulty based on overt criteria - which correlate with such a distinction. Instead, the authors later clarify that the dissociation is between a verbal circuit and a quantity circuit (Dehaene et al. 2003). In an extension of this, Tschentscher and Hauk (2014) evidence that the functional networks recruited during calculation vary as a function of individual strategy (recalling from memory versus calculating a solution), and that these networks mirror those identified when more classic distinctions between surface complexity (i.e., easy versus complex arithmetic problems) are made. Their results indicate that caution is required when interpreting some of the prior neuroimaging work, where surface complexity has been confounded with strategy. In addition, this highlights that variation in the neural systems engaged for arithmetic problem solving will be reflective of individual differences in knowledge of arithmetic facts, as variation in individual knowledge will dictate the strategies engaged, and thus the brain systems used, to solve numerical problems. Fact retrieval may be more common for certain operands and problem structures, but this is likely to reflect individual education or training, rather than there being a blanket cognitive process associated with specific operands per se. Indeed, cross-sectional work demonstrates a developmental shift in neural processing that is indicative of increasing reliance on verbal retrieval in multiplication and quantity-based procedures for subtraction, as a function of educational stage (Prado et al. 2014). On a broader scale, what this implies is that the TCM is not applicable in all instances, for example, in the case of infants and young children, and for children and adults with developmental disorders. In the case of typical children, it has been argued that one could use the model in order to examine how the 'end point' of numerical proficiency is achieved. 
There are, nevertheless, differences in how the brain is recruited for numerical cognition by children versus adults ${ }^{1}$, and that this evolves as a function of age, experience and ability (Emerson \& Cantlon 2015). Conversely, much less is known about the neurodevelopmental bases of mathematical learning disability and developmental dyscalculia. Whilst neuropsychological case studies can generally attribute behavioural difficulties that result from damage to an otherwise normally developed brain region, in atypical development, that same brain region may function atypically due to different development of other brain regions that would ordinarily support typical functional specialisation (see Ansari, 2010 for a detailed discussion). In support of this perspective, a meta-analysis has demonstrated that the pattern of brain activation in number-related tasks differs between children with dyscalculia and typically developing children, in terms of both the magnitude and foci of activation (Kaufmann et al. 2011). Differences in brain structure have also been identified (e.g., Molko et al. 2003; Rykhlevskaia et al. 2009). Lastly, behavioural work evidences that numerical tasks are approached differently in children with dyscalculia, versus typical controls (e.g., Mazzocco et al. 2011a). In sum, developmental dyscalculia presents differently in terms of neural development, and structural and functional anatomy, as well as the cognitive phenotype. Considered together, these data suggest that the use of findings from adult neuropsychology are not appropriate to developmental disorders (Karmiloff-Smith 1998).

\section{Neurocognitive Features of Number}

\section{Modes of Representation: Non-Symbolic and Symbolic Number}

As touched upon earlier in this chapter, the form in which cardinal number can be conveyed -

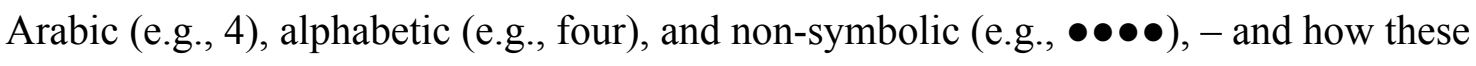
formats might be used to identify any specific number area(s) in the brain, has been the focus

\footnotetext{
${ }^{1}$ For a developmental perspective on calculation, interested readers may wish to view Kaufmann, Wood, Rubinsten, and Henik (2011)
} 
of much interest in Cognitive Neuroscience. Understanding how the brain represents number has translated into numerous functional imaging studies, where the neural correlates of symbolic versus non-symbolic number have been explored extensively. In particular, this endeavour can be characterised by an overarching interest in whether to consider the representation of number as abstract - that is, independent of the notational format (termed notation independent) - or non-abstract - accordingly, notation dependant. Thus, for an abstract (i.e. notation independent) representation of the numerical information, neuronal populations that code the magnitude 7 would make no distinction between "7" and "seven" (Cohen Kadosh \& Walsh 2009).

Fias et al. (2003) used PET in one of the first attempts using functional neuroimaging to identify an abstract representation of magnitude. Participants were asked to complete a magnitude comparison task featuring pairs of angles (size), lines (length), or Arabic digits (quantity). By taking the conjunction of these, and contrasting the resulting neural activation with that from a control condition requiring luminance judgements about the same stimuli, the left IPS demonstrated a notation independent response. The large spatial smoothing kernel that is applied to increase the signal-to-noise ratio in PET studies (and fMRI, to a lesser degree), however, can result in the blurring of activations. This is particularly problematic if neurons with different encoding properties are intermingled within a single cortical area, as has been demonstrated in primates (Tudusciuc \& Nieder 2007). Accordingly, caution should be exercised in interpreting these results as indicative of a domain specific number module. Evidence for number-selective neuronal populations has also been sought using the fMRI adaptation (fMRIa) paradigm. Underpinned by the premise that repetition suppression will determine response specificity, fMRIa enables one to quantify the selectivity of a population of neurons on a scale that is much smaller than the volume of a voxel (see Krekelberg et al. 2006 for theorethical discussion). As adaptation can be achieved through passive viewing, fMRIa can negate the need for an overt behavioural response, therefore 
removing any confounding effects that are typically associated with classic stimulus-response paradigms. Using this approach, Piazza et al. (2007) showed that the parietal cortex coded for magnitude, independent of form - where, by taking the peak voxel within IPS of each participant, they identified a pattern of signal change that was characteristic of adaptation. However, they also observed an interaction effect between recovery and form in the left IPS, suggesting notation dependent processing was occurring. A similar study also using fMRIa yielded only notation dependent responses in the IPS (Cohen Kadosh, Cohen Kadosh, Kaas, et al. 2007). TMS has also evidenced notation-dependent numerical representation in the IPS (Cappelletti et al. 2007; Cohen Kadosh et al. 2010).

The seemingly incompatible findings between, for example, Piazza et al. (2002) and Cohen Kadosh et al. (2007) highlight some of the challenges faced by researchers in this area. Conflicting results could be as a result of differences in experimental design. In this case, the two studies differed in terms of the stimuli, where the former used dot patterns and Arabic digits, and the latter used alphabetic number-words and Arabic digits. Regardless, as stated earlier, for the definition of an abstract representation of number to hold, these paradigmatic differences should not preclude the activation of neurons that care only about magnitude; therefore, both results should have demonstrated adaptation following the change of notation. Of course, it is commonly argued that an absence of evidence is not evidence for absence, making the task of effectively demonstrating a null result theoretically challenging. Lack of statistical power is one reason for a null result, for example. Regardless, note that the oftquoted loss of power due to inter-subject variation ${ }^{2}$ in functional neuroanatomy would not affect either study here, as both analyses reflected data taken from coordinates in singlesubject space. Secondly, the two studies differed regarding pre-processing, most crucially, the use of spatial smoothing. Spatial smoothing was applied by Piazza et al. (2007) but not Cohen Kadosh et al.(Cohen Kadosh, Cohen Kadosh, Kaas, et al. 2007). As discussed

\footnotetext{
${ }^{2}$ There are, of course, many other potential causes
} 
beforehand, spatial smoothing is beneficial in certain circumstances, and is considered helpful in increasing signal detection whilst removing noise. Notwithstanding, it can affect localization, by muddying activation within intermingled populations of neurons or where cluster peaks are in spatially proximal locations. One solution that will address some of these challenges is to use multivariate statistics, which can be more sensitive than fMRIa (Sapountzis et al. 2010). For example, machine learning approaches, such as multivoxel pattern analysis (MVPA), offer increased capacity to detect spatial patterns of activation that can discriminate between discrete categories of stimuli. Eger et al (2009) used MVPA to examine whether a pattern classification algorithm trained to the specific numerosity of dot arrays could be used to discriminate the semantic value of Arabic numbers, and vice versa. Whilst classifiers trained on numerosity for non-symbolic numbers were good at discriminating within-format numerosities, performance was poor or below chance when applied to symbolic number. This asymmetrical response across number formats suggests that, whilst parts of the IPS work together to return magnitude information, the evidence for notation independent neurons in this case is weak. Indeed, other MVPA studies support this conclusion by further demonstrating poor generalisation across notations (Damarla \& Just 2013; Bulthé et al. 2014; Bulthé et al. 2015).

It has been suggested that IPS activity may correlate with the number of individual visual objects in a stimulus, rather than the magnitude per se (Cohen 2009; Bulthé et al. 2015). Thus, if physical similarity between stimuli in magnitude comparison underpins quantity representation, behavioural and neural outcomes should be predicted by a measure of the degree of physical similarity between a distractor and target. By computing a function of physical similarity between a target Arabic numeral (in this case, 5; Figure 6) and all other single digits, Cohen (2009) highlighted that the degree of visual similarity between the target and distractor numeral was a stronger predictor of reaction time than a function of the numerical distance between them. Moreover, the predictive value of physical distance was 
not significant if both visual similarity and physical distance were included in the model, suggesting that Arabic numerals maintain a level of independence from the quantity that they represent. This result has been recently demonstrated in IPS using MVPA (Bulthé et al. 2015). Here, the authors argue that studies of number representation often use a font for Arabic numerals that comprises one unit: the physical shape of the digit is a single line. It would therefore be the shape, not the quantity, of visual elements that ascribe semantic meaning of Arabic numerals in this format. Note that this format is in contrast to what was used in Cohen (2009), where Arabic numerals were presented in an analogue font; thus, each digit comprised a number of lines to form the shape of a number (e.g., 8, as depicted in Figure 6 comprises 7 distinct objects). Using Representational Similarity Analysis (RSA), a machine-learning method used to compute similarities across neural responses (Kriegeskorte, Mur, \& Bandettini, 2008), Bulthé et al. (2015) were able to examine classification patterns for individual magnitudes. Here, a pattern classification algorithm was trained to differentiate different numerosities of dot arrays. When applied to Arabic digits, however, the classifier made no distinction for numerical magnitude. Instead, it classified all digits as a one-dot pattern, reflecting that there was indeed one object - the Arabic digit - in the visual scene. This, the authors' argue, is inconsistent with the view that numerical coding in IPS is notation independent. Likewise, using RSA, Lyons et al. (2015) demonstrate that the correlation between activity in IPS in pairs of cross-format numbers (matched in numerosity) were indicative of differences in not only representational structure, but the representations themselves.

\author{
$<$ FIGURE 6 ABOUT HERE $>$ \\ <FIGURE 6 FROM: Cohen (2009).>
}

The evidence outlined highlights some of the complexities of studying numerical cognition in the brain. The current literature suggests that IPS is fundamental in the case of 
representing magnitude, but that there are differences in the way that this region processes number, which depend on notation (Cohen Kadosh \& Walsh 2009; Bulthé et al. 2014; Bulthé et al. 2015; Lyons et al. 2015). Certainly, RSA has facilitated close inspection of the neural signature of specific numerosities, revealing qualitative differences in symbolic and nonsymbolic number (Bulthé et al. 2015; Lyons et al. 2015). Nevertheless, there is general consensus that IPS is sensitive to numerosity. The minutiae, however, are still very much debated (see Piazza \& Eger, 2015 for further review).

\section{Neurocognitive Features of Number}

\section{Processing of Quantity: An Approximate Number \& Object Tracking System}

The Approximate Number System, as outlined earlier, is a frequently posited pre-verbal system for numerical quantification (see reviews, Piazza 2010; Hyde 2011; Hyde \& Spelke 2011). In contrast to a discrete representation of numerical values, the Approximate Number System is suggested to facilitate a degree of quantification that is analogous to estimating. Neuroimaging studies seeking to identify the neural bases for an Approximate Number System have typically used passive fixation, numerosity comparison, or approximate calculation using dot arrays. For example, Izard et al. (2008) used a passive adaptation paradigm whilst acquiring EEG data from 3-month-old infants. By comparing the eventrelated potentials (ERPs) following presentation of the habituated numerosity versus a sparse, deviant numerosity, the authors identified a frontoparietal network that was spatiotemporally distinct to a control task involving shape change. This process has been more precisely localised to the right IPS with 6-month-old infants, using fNIRS and a similar adaptation paradigm (Hyde et al. 2010). Furthermore, cross-sectional fMRI has demonstrated a significant overlap in the recruitment of right IPS during numerical approximation between 4year-old children and adults (Cantlon et al. 2006). Together, these data are indicative of a potential primitive system for the representation of magnitude (numerical or non-numerical 
sensory cues) in the right IPS, and that this system is present from early infancy and, to some degree, consistent in adulthood.

Earlier in this chapter, we outlined the ratio-dependent effect as a characteristic signature of the Approximate Number System, where the discriminability between two sets depends on their numerical ratio (Weber's law). Work with non-human primates has demonstrated neural tuning curves that are consistent with this formulation (see Nieder, 2013 for a review). Single unit recording highlights the existence of neurons that demonstrate a 'preferred' numerosity in the prefrontal and posterior parietal cortices (Nieder et al. 2002; see figure 7). Importantly, for each cell's preferred numerosity, a progressive reduction in activity was found for numerosities as a function of numerical distance. Thus, a neuron that was maximally active for the number 3 , for example, was less responsive to the numbers 2 and 4 , with the overall lowest response reflecting the array with the smallest cardinal value (in this case, 2; see green line figure 7B). The neurons consecutively arranged overlapping tuning curves retained an inherent order of cardinalities, allowing for numerosities to exist in relation to one another, thus reflecting meaningful quantity information. Later work (Nieder \& Miller 2004) demonstrated that, whilst similar properties were exhibited by neurons in the prefrontal and posterior parietal cortex, those in the posterior parietal cortex were responsive to numerosity sooner than those in the prefrontal cortex. A wider expanse of cortex was dedicated to this process in the prefrontal cortex and the tuning of neurons in this region was stronger. The authors conclude that quantity is initially extracted by neurons within the posterior parietal cortex, then fed forward and expanded in the prefrontal cortex to support online executive processes.

$<$ FIGURE 7 ABOUT HERE $>$

<FIGURE 7 FROM: Andreas Nieder and Miller (2004).> 
Piazza et al. (2004) sought to confirm whether the bilateral IPS encoded numerical quantity, and that activation in this region also followed Weber's law. Using a passive fMRI task, the authors demonstrated that the horizontal IPS was responsive to non-symbolic numerosity. Though noisy, by extracting the level of activity from the peak voxel in each subject's data set, the authors were able to generate curves that were comparable to psychophysical curves extracted from the same sample. Moreover, both behavioural and brain data were in line with Weber's law, providing strong evidence for an Approximate Number System.

The incidence of this system for rapid quantity estimation has been suggested to indicate an evolutionary basis for elementary arithmetic in humans (Nieder 2013)(A. Nieder, 2013)(A. Nieder, 2013). From this, one might anticipate that the representation of symbolic number may piggyback neural systems for non-symbolic representation. A recent study using RSA, however, demonstrated that there was no relationship between the tuning curves in bilateral IPS for non-symbolic (dot arrays) and symbolic numbers in human adults (Lyons et al. 2015). Whilst the IPS demonstrated the more classic, overlapping pattern of tuning curves in pairs of non-symbolic numerosities, activation for pairs of Arabic numerals reflected a more discrete representation, with no systematic overlap between numbers. Considering the evidence reviewed in sum, comparative, behavioural and neurophysiological evidence points to the IPS for quantisation - an Approximate Number System. Early evidence from human adults, however, indicates qualitative differences in how IPS supports estimation for symbolic versus non-symbolic number (Lyons et al. 2015).

The Object Tracking System is a mechanism by which objects are represented as distinct entities. Said to have a capacity limited to around 3 or 4 items, the Object Tracking System is characterised by the facility to rapidly enumerate the precise quantity of elements 
within an $\operatorname{array}^{3}$ (Mazza \& Caramazza 2015). Whilst the occurrence of subitizing is not in itself disputed, the degree to which the Approximate Number System might support estimation of small sets (i.e. sets that are within the subitizing range), or in what circumstances the Object Tracking System might take over, and whether these systems are dissociable, has been widely debated. Early neuroimaging studies that sought to contrast small versus larger set sizes were indicative of a single system, where regions that support enumeration of small arrays were shown to be activated similarly than when enumerating larger arrays (Piazza et al. 2002). This led some researchers to suggest that the Approximate Number System operates over the entire range of numbers: a "one system view" (see Hyde et al. 2010 who review evidence for the "one system view"). Recent work, however, converges to suggest that set size does indeed affect encoding, although the neural systems are still not well established. For example, ERP data demonstrate a distinction between small and larger numerical quantities that would be outside of the subitizing range (Hyde \& Spelke 2009). Evidence for reduced response latencies for arrays within the subitizing range has been demonstrated in the posterior temporal cortex using MEG (Vuokko et al. 2013). Cutini et al. (2014) used an event-related enumeration task and fNIRS in an attempt to dissociate subitizing from estimation. Participants were given 200 milliseconds to view and subsequently articulate the precise quantity of a dot array, with arrays ranging in size from 2 to 6 elements per trial - thus, crossing the classic range from subitizing to estimation. By contrasting activation with a control condition featuring Arabic stimuli within the same range, the authors identified a right dominant, parieto-occipito network. Importantly, bilateral IPS was modulated by numerical magnitude, wherein a sigmoidal function best explained the relationship between magnitude and brain response. A sharp increase in activation was seen when comparing the neural response between numerosities of 4 and 5, and there was a lag in

\footnotetext{
${ }^{3}$ Note that the term subitizing describes the precise enumeration of small quantities and is distinguished from counting
} 
the haemodynamic response for arrays outside of the subitizing range, indicating an altogether different neural time course when enumerating small versus larger arrays. With fMRIa, Demeyere et al. (2014) demonstrated a "number specific repetition effect" in the subitizing range which was associated with additional processing in the left occipito-temporo cortex.

The evidence reviewed is indicative of an additional neural system which may support the rapid enumeration of small quantities. Recent behavioural work, however, suggests that the occurrence of subitizing is context specific. Working on the basis that absence of the ratio-dependent effect is indicative of Object Tracking System activation, Agrillo et al. (2015) demonstrated that it was the presence (or absence) of task-irrelevant stimuli in the visual field that determined ratio-dependence in small numerosities. By comparing a number of classic experimental formats for dot comparison tasks, the authors demonstrated that when the sum total of dots was greater than the subitizing amount, the ratio-dependent effect was observed. This is in line with proponents of the "two system view", who suggest that attentional load modulates the engagement of the Object Tracking System and Approximate Number System, where items presented outside of attentional limits cannot be represented as individual units (Hyde, 2011).

\section{General Concluding Comments}

In this chapter, we have outlined research from Developmental and Experimental Psychology, and Cognitive Neuroscience. Most of the studies have focussed on symbolic and non-symbolic numerical processing. One of the core areas of interest in prior work has been whether non-symbolic processing contributes to the development of symbolic processing. However, the potential confounding influence of non-numerical features on non-symbolic numerical processing is still a matter for debate. Regarding symbolic numerical processing, 
studies have focussed on the acquisition of the symbolic system through counting, and how non-symbolic representation is mapped onto an arbitrary, culturally determined, symbolic system. Researchers have also explored symbolic processing and its interaction with space and other magnitudes. Studies of the human and animal brain have demonstrated a core network for representing quantity, with particular focus on the IPS and prefrontal cortex. With development, some brain areas appear to become specialised in processing numerical information depending on the format: non-symbolic representation has been localised primarily to the IPS, the process of verbal representation to the angular gyrus, and temporal regions to support visual Arabic processing. One suggestion is that humans are born equipped with a basic system that is devoted to magnitudes in general. With exposure to numerical stimuli within the environment and interaction with symbolic systems, neural circuits dedicated to assimilating numerical information develop through a process of Interactive Specialisation (Cohen Kadosh \& Johnson 2007; Cohen Kadosh \& Walsh, 2008; Johnson 2001). Whilst the chapter has been primarily focused on numerical cognition, we also briefly outlined the contribution of basic numerical processes and other cognitive functions to mathematical achievement. Whilst mathematical achievement will be of interest to a broad readership, we see mathematical achievement as the resultant outcome culminating from an array of numerical, cognitive and socio-emotional factors. Moreover, the relative weighting of these factors in terms of their contribution to mathematical achievement can change across time. For instance, basic numerical representation can have a larger influence on mathematical achievement in the early years of education when children are developing solid links between non-symbolic, symbolic and verbal representation. Conversely, in the later stages of education, factors like logic and reasoning can have a stronger influence on mathematical achievement. Consequently, mathematical achievement is used to describe what is actually an evolving construct. However, this should not detract from research seeking to understand how basic numerical skills relate to later mathematical performance. 
The complex interplay between numerical cognition, neural development, education and mathematical achievement, however, has yet been well characterised. Future studies should seek to examine this using a longitudinal approach wherein the broader cognitive, social and emotional features are coupled with developing mathematical achievement. 


\section{References}

Agrillo, C. et al., 2015. Ratio dependence in small number discrimination is affected by the experimental procedure. Frontiers in Psychology, 6(OCT), pp.1-13.

Algom, D., Dekel, A. \& Pansky, A., 1996. The perception of number from the separability of the stimulus: the Stroop effect revisited. Memory \& cognition, 24(5), pp.557-572.

Andres, M., Michaux, N. \& Pesenti, M., 2012. Common substrate for mental arithmetic and finger representation in the parietal cortex. NeuroImage, 62(3), pp.1520-1528. Available at: http://dx.doi.org/10.1016/j.neuroimage.2012.05.047.

Ansari, D., 2010. Neurocognitive approaches to developmental disorders of numerical and mathematical cognition: The perils of neglecting the role of development. Learning and Individual Differences, 20(2), pp.123-129. Available at: http://www.sciencedirect.com/science/article/pii/S1041608009000454.

Antell, S.E. \& Keating, D.P., 1983. Perception of numerical invariance in neonates. Child development, 54(3), pp.695-701.

Arsalidou, M. \& Taylor, M.J., 2011. Is 2+2=4? Meta-analyses of brain areas needed for numbers and calculations. NeuroImage, 54(3), pp.2382-2393. Available at: http://www.sciencedirect.com/science/article/pii/S1053811910013017 [Accessed January 21, 2014].

Arsalidou, M., \& Taylor, M. J. (2011). Is 2+2=4? Meta-analyses of brain areas needed for numbers and calculations. NeuroImage, 54(3), 2382-2393.

Ashcraft, M.H. \& Fierman, B. a, 1982. Mental addition in third, fourth, and sixth graders. Journal of Experimental Child Psychology, 33(2), pp.216-234.

Ashcraft, M.H., 1982. The development of mental arithmetic: A chronometric approach. Developmental Review, 2, pp.213-236.

Barth, H.C. \& Paladino, A.M., 2011. The development of numerical estimation: evidence against a representational shift. Developmental science, 14(1), pp.125-35. Available at: 
http://www.ncbi.nlm.nih.gov/pubmed/21159094Spence, [Accessed February 18, 2011].

Bates, E. et al., 2003. Voxel-based lesion-symptom mapping. Nat Neurosci, 6(5), pp.448450. Available at: http://dx.doi.org/10.1038/nn1050.

Bencini, G.M.L. et al., 2011. When two and too don't go together: A selective phonological deficit sparing number words. Cortex, 47(9), pp.1052-1062. Available at: http://www.sciencedirect.com/science/article/pii/S0010945211000773.

Berch, D.B. et al., 1999. Extracting parity and magnitude from Arabic numerals: developmental changes in number processing and mental representation. Journal of experimental child psychology, 74(4), pp.286-308. Available at: http://www.ncbi.nlm.nih.gov/pubmed/10552920.

Berger, A., Tzur, G., \& Posner, M. I. (2006). Infant brains detect arithmetic errors. Proc Natl Acad Sci U S A, 103(33), 12649-12653. doi:10.1073/pnas.0605350103

Berteletti, I. et al., 2010. Numerical estimation in preschoolers. Developmental psychology, 46(2), pp.545-51. Available at: http://www.ncbi.nlm.nih.gov/pubmed/20210512.

Berteletti, I., Lucangeli, D. \& Zorzi, M., 2012. Representation of numerical and nonnumerical order in children. Cognition, 124(3), pp.304-313. Available at: http://dx.doi.org/10.1016/j.cognition.2012.05.015.

Booth, J.L. \& Siegler, R.S., 2006. Developmental and individual differences in pure numerical estimation. Developmental psychology, 42(1), pp.189-201. Available at: http://www.ncbi.nlm.nih.gov/pubmed/16420128.

Booth, J.L. \& Siegler, R.S., 2008. Numerical magnitude representations influence arithmetic learning. Child development, 79(4), pp.1016-31. Available at: http://www.ncbi.nlm.nih.gov/pubmed/18717904.

Briars, D. \& Siegler, R.S., 1984. A featural analysis of preschoolers' counting knowledge. Developmental Psychology, 20(4), pp.607-618.

Brysbaert, M., Fias, W. \& Reynvoet, B., 2002. Semantic priming in number naming. , 
(August 2015). Available at:

http://www.informaworld.com/smpp/title $\sim$ content $=\mathrm{g} 713932604 \sim \mathrm{db}=\mathrm{all}$.

Brysbaert, M., Fias, W., et al., 2002. Semantic priming in number naming. The quarterly journal of experimental psychology, 55A(4), pp.1127-1139. Available at: http://www.informaworld.com/smpp/title $\sim$ content $=\mathrm{g} 713932604 \sim \mathrm{db}=$ all.

Bueti, D. \& Walsh, V., 2009. The parietal cortex and the representation of time, space, number and other magnitudes. Philosophical transactions of the Royal Society of London. Series B, Biological sciences, 364(1525), pp.1831-40.

Bugden, S. \& Ansari, D., 2011. Individual differences in children's mathematical competence are related to the intentional but not automatic processing of Arabic numerals. Cognition, 118(1), pp.32-44. Available at: http://www.ncbi.nlm.nih.gov/pubmed/20970782 [Accessed May 4, 2014].

Bulthé, J., De Smedt, B. \& Op de Beeck, H.P., 2014. Format-dependent representations of symbolic and non-symbolic numbers in the human cortex as revealed by multi-voxel pattern analyses. NeuroImage, 87, pp.311-322.

Bulthé, J., De Smedt, B. \& Op de Beeck, H.P., 2015. Visual Number Beats Abstract Numerical Magnitude: Format-dependent Representation of Arabic Digits and Dot Patterns in Human Parietal Cortex. Journal of Cognitive Neuroscience, 27(7), pp.13761387. Available at: http://dx.doi.org/10.1162/jocn_a_00787.

Burr, D.C. \& Ross, J., 2008. A visual sense of number. Current biology : CB, 18(6), pp.4258. Available at: http://www.ncbi.nlm.nih.gov/pubmed/18342507 [Accessed January 29, 2013].

Burr, D.C., Turi, M. \& Anobile, G., 2010. Subitizing but not estimation of numerosity requires attentional resources. Journal of Vision, 10(6), pp.1-10.

Butterworth, B., 2010. Foundational numerical capacities and the origins of dyscalculia. Trends in cognitive sciences, 14(12), pp.534-541. Available at: 
http://www.ncbi.nlm.nih.gov/pubmed/20971676 [Accessed October 28, 2010].

Butterworth, B., Cappelletti, M. \& Kopelman, M., 2001. Category specificity in reading and writing: the case of number words. Nat Neurosci, 4(8), pp.784-786.

Cantlon, J.F. et al., 2006. Functional imaging of numerical processing in adults and 4-y-old children. Plos Biology, 4(5), pp.844-854. Available at: $<$ Go to ISI $>$ ://WOS:000237966900021.

Cantlon, J.F., Platt, M.L. \& Brannon, E.M., 2009. Beyond the number domain. Trends Cogn Sci, 13(2), pp.83-91.

Cappelletti, M. et al., 2007. rTMS over the intraparietal sulcus disrupts numerosity processing. Experimental Brain Research, 179(4), pp.631-642.

Carey, S., 2001. Cognitive Foundations of Arithmetic: Evolution and Ontogenisis. Mind and Language, 16(1), pp.37-55.

Carey, S., 2009. Where Our Number Concepts Come From. Journal of Philosophy, 106(4), pp. $220-254$.

Castronovo, J. \& Seron, X., 2007. Numerical estimation in blind subjects: evidence of the impact of blindness and its following experience. Journal of experimental psychology. Human perception and performance, 33(5), pp.1089-106. Available at: http://www.ncbi.nlm.nih.gov/pubmed/17924809 [Accessed September 3, 2014].

Caviola, S. et al., 2012. The involvement of working memory in children's exact and approximate mental addition. Journal of Experimental Child Psychology, 112(2), pp.141-160. Available at: http://dx.doi.org/10.1016/j.jecp.2012.02.005.

Cipolotti, L., Butterworth, B. \& Denes, G., 1991. A specific deficit for numbers in a case of dense acalculia. Brain, 114, pp.2619-2637. Available at: $<$ Go to ISI>://WOS:A1991HC61100015.

Cipolotti, L., Warrington, E.K. \& Butterworth, B., 1995. Selective Impairment in Manipulating Arabic Numerals. Cortex, 31(1), pp.73-86. Available at: 
http://www.sciencedirect.com/science/article/pii/S0010945213801062.

Cipora, K. \& Nuerk, H.-C., 2013. Is the SNARC effect related to the level of mathematics? No systematic relationship observed despite more power, more repetitions, and more direct assessment of arithmetic skill. Quarterly journal of experimental psychology (2006), 66(March), pp.1974-91. Available at:

http://www.ncbi.nlm.nih.gov/pubmed/23473520.

Cipora, K. et al., 2015. Professional mathematicians differ from controls in their spatialnumerical associations. Psychological Research. Available at: http://link.springer.com/10.1007/s00426-015-0677-6.

Clayton, S. \& Gilmore, C., 2014. Inhibition in dot comparison tasks. Zdm, pp.1-12. Available at: http://dx.doi.org/10.1007/s11858-014-0655-2 [Accessed December 18, 2014].

Clearfield, M.W. \& Mix, K.S., 1999. Number Versus Contour Length in Infants' Discrimination of Small Visual Sets. Psychological science, 10(5), pp.408-411.

Clearfield, M.W. \& Mix, K.S., 2001. Amount Versus Number : Infants' Use of Area and Contour Length to Discriminate Small Sets. Journal of Cognition and Development, 2(3), pp.243-260.

Cohen Kadosh, K. \& Johnson, M.H., 2007. Developing a cortex specialized for face perception. Trends in Cognitive Sciences, 11(9), pp.367-369.

Cohen Kadosh, R. \& Henik, A., 2006. A Common Representation for Semantic $n$ nand Physical Properties. Experimental Psychology (formerly “Zeitschrift für Experimentelle Psychologie”), 53(2), pp.87-94. Available at:

http://psycontent.metapress.com/openurl.asp?genre=article\&id=doi:10.1027/16183169.53.2.87.

Cohen Kadosh, R. \& Walsh, V., 2009. Numerical representation in the parietal lobes: abstract or not abstract? The Behavioral and brain sciences, 32(3-4), pp.313-28-73. Available at: 
http://www.ncbi.nlm.nih.gov/pubmed/19712504.

Cohen Kadosh, R. et al., 2010. Double dissociation of format-dependent and number-specific neurons in human parietal cortex. Cereb Cortex, 20(9), pp.2166-2171.

Cohen Kadosh, R., \& Dowker, A. (2015). The Oxford Handbook of Numerical Cognition: OUP Oxford.

Cohen Kadosh, R., \& Walsh, V. (2008). From magnitude to natural numbers: A developmental neurocognitive perspective. Behavioral and Brain Sciences, 31, 647-648.

Cohen Kadosh, R., Cohen Kadosh, K., Kaas, A., et al., 2007. Notation-Dependent and Independent Representations of Numbers in the Parietal Lobes. Neuron, 53(2), pp.307314. Available at: http://dx.doi.org/10.1016/j.neuron.2006.12.025.

Cohen Kadosh, R., Cohen Kadosh, K., Linden, D.E.J., et al., 2007. The Brain Locus of Interaction between Number and Size : A Combined Functional Magnetic Resonance Imaging and Event-related Potential Study. , pp.957-970.

Cohen Kadosh, R., Cohen Kadosh, K., Schuhmann, T., et al., 2007. Virtual dyscalculia induced by parietal-lobe TMS impairs automatic magnitude processing. Current biology : CB, 17(8), pp.689-693. Available at: <Go to ISI>://MEDLINE:17379521.

Cohen Kadosh, R., Lammertyn, J. \& Izard, V., 2008. Are numbers special? An overview of chronometric, neuroimaging, developmental and comparative studies of magnitude representation. Progress in Neurobiology, 84(2), pp.132-147.

Cohen, D.J., 2009. Integers do not automatically activate their quantity representation. Psychonomic bulletin \& review, 16(2), pp.332-336. Available at: http://www.ncbi.nlm.nih.gov/pmc/articles/PMC2658745/.

Crollen, V. \& Noël, M., 2015. The role of fi ngers in the development of counting and arithmetic skills. Acta Psychologica, 156, pp.37-44. Available at: http://dx.doi.org/10.1016/j.actpsy.2015.01.007.

Crollen, V., Castronovo, J. \& Seron, X., 2011. Under- and over-estimation: a bi-directional 
mapping process between symbolic and non-symbolic representations of number? Experimental psychology, 58(1), pp.39-49. Available at:

http://www.ncbi.nlm.nih.gov/pubmed/20494869 [Accessed September 3, 2014].

Cui, X. et al., 2011. A quantitative comparison of NIRS and fMRI across multiple cognitive tasks. NeuroImage, 54(4), pp.2808-2821.

Cutini, S., Scarpa, F., et al., 2014. Number-space interactions in the human parietal cortex: Enlightening the SNARC effect with functional near-infrared spectroscopy. Cereb Cortex, 24(2), pp.444-451.

Cutini, S., Scatturin, P., et al., 2014. Are the neural correlates of subitizing and estimation dissociable? An fNIRS investigation. NeuroImage, 85 Pt 1, pp.391-399.

Damarla, S.R. \& Just, M.A., 2013. Decoding the representation of numerical values from brain activation patterns. Hum Brain Mapp, 34(10), pp.2624-2634.

de Hevia, M.D. \& Spelke, E.S., 2010. Number-Space Mapping in Human Infants. Psychological Science, 21(5), pp.653-660. Available at: http://pss.sagepub.com/lookup/doi/10.1177/0956797610366091.

De Smedt, B. \& Gilmore, C.K., 2011. Defective number module or impaired access? Numerical magnitude processing in first graders with mathematical difficulties. Journal of experimental child psychology, 108(2), pp.278-92. Available at: http://www.sciencedirect.com/science/article/pii/S0022096510001773.

De Smedt, B. et al., 2013. How do symbolic and non-symbolic numerical magnitude processing skills relate to individual differences in children's mathematical skills? A review of evidence from brain and behavior. Trends in Neuroscience and Education, pp.1-8.

Deary, I.J. et al., 2007. Intelligence and educational achievement. Intelligence, 35(1), pp.1321.

Dehaene, S. \& Cohen, L., 1995. Towards an anatomical and functional model of number 
processing. Mathematical cognition, 1(1), pp.83-120.

Dehaene, S. \& Cohen, L., 1997. Cerebral pathways for calculation: double dissociation between rote verbal and quantitative knowledge of arithmetic. Cortex; a journal devoted to the study of the nervous system and behavior, 33(2), pp.219-250.

Dehaene, S. (2003). The neural basis of the Weber-Fechner law: a logarithmic mental number line. Trends Cogn Sci, 7(4), 145-147.

Dehaene, S. et al., 2003. Three Parietal Circuits for Number Processing. Cognitive Neuropsychology, 20(3-6), pp.487-506. Available at: http://www.informaworld.com/openurl?genre=article\&doi=10.1080/0264329024400023 9\&magic $=$ crossref $\mid$ D404A21C5BB053405B1A640AFFD44AE3 [Accessed July 26, 2010].

Dehaene, S., \& Cohen, L. (1997). Cerebral Pathways for Calculation: Double Dissociation between Rote Verbal and Quantitative Knowledge of Arithmetic. Cortex, 33(2), 219250. doi:http://dx.doi.org/10.1016/S0010-9452(08)70002-9

Dehaene, S., 2003. The neural basis of the Weber-Fechner law: a logarithmic mental number line. Trends in cognitive sciences, 7(4), pp.145-147. Available at: http://www.ncbi.nlm.nih.gov/pubmed/12691758.

Dehaene, S., 2011. The Number Sense: How the Mind Creates Mathematics, Revised and Updated Edition,

Dehaene, S., Bossini, S. \& Giraux, P., 1993. The mental representation of parity and number magnitude. Journal of Experimental Psychology: General, 122(3), pp.371-396.

Delazer, M. \& Benke, T., 1997. Arithmetic facts without meaning. Cortex, 33, pp.697-710.

Delazer, M. et al., 2002. When writing 0 (zero) is easier than writing O (o): a neuropsychological case study of agraphia. Neuropsychologia, 40(12), pp.2167-2177. Available at: http://www.sciencedirect.com/science/article/pii/S0028393202000441. Delazer, M. et al., 2005. Learning by strategies and learning by drill-evidence from an fMRI 
study. Neuroimage, 25(3), pp.838-849.

Demeyere, N., Rotshtein, P. \& Humphreys, G.W., 2014. Common and dissociated mechanisms for estimating large and small dot arrays: Value-specific fMRI adaptation. Human Brain Mapping, 35(8), pp.3988-4001. Available at: http://dx.doi.org/10.1002/hbm.22453.

DeStefano, D. \& LeFevre, J., 2004. The role of working memory in mental arithmetic. European Journal of Cognitive Psychology, 16(3), pp.353-386.

DeWind, N.K. et al., 2015. Modeling the approximate number system to quantify the contribution of visual stimulus features. Cognition, 142, pp.247-265. Available at: http://linkinghub.elsevier.com/retrieve/pii/S0010027715001134.

Doricchi, F. et al., 2005. Dissociation between physical and mental number line bisection in right hemisphere brain damage. Nat Neurosci, 8(12), pp.1663-1665.

Ebersbach, M. \& Erz, P., 2014. Symbolic versus non-symbolic magnitude estimations among children and adults. Journal of experimental child psychology, 128C, pp.52-68. Available at: http://www.ncbi.nlm.nih.gov/pubmed/25077407 [Accessed August 5, 2014].

Ebersbach, M. et al., 2008. The relationship between the shape of the mental number line and familiarity with numbers in 5- to 9-year old children: evidence for a segmented linear model. Journal of experimental child psychology, 99(1), pp.1-17.

Eger, E. et al., 2009. Deciphering Cortical Number Coding from Human Brain Activity Patterns. Current Biology, 19(19), pp.1608-1615. Available at: http://www.sciencedirect.com/science/article/pii/S0960982209016236.

Eger, E. et al., 2013. Spatially Invariant Coding of Numerical Information in Functionally Defined Subregions of Human Parietal Cortex. Cereb Cortex, (May), pp.1319-1329. Available at: http://www.ncbi.nlm.nih.gov/pubmed/24293562.

Emerson, R.W. \& Cantlon, J.F., 2015. Continuity and change in children's longitudinal 
neural responses to numbers. Developmental science, 18(2), pp.314-326. Available at: http://dx.doi.org/10.1111/desc.12215.

Fantz, R.L., 1964. Visual Experience in Infants: Decreased Attention To Familiar Patterns Relative To Novel Ones. Science (New York, N.Y.), 146, pp.668-670.

Feigenson, L., Carey, S. \& Hauser, M., 2002. The representations underlying infants' choice of more: object files versus analog magnitudes. Psychological science : a journal of the American Psychological Society / APS, 13(2), pp.150-6. Available at: http://www.ncbi.nlm.nih.gov/pubmed/11933999.

Feigenson, L., Carey, S. \& Spelke, E., 2002. Infants' discrimination of number vs. continuous extent. Cognitive psychology, 44(1), pp.33-66. Available at: http://www.ncbi.nlm.nih.gov/pubmed/11814309 [Accessed September 23, 2010].

Feigenson, L., Dehaene, S. \& Spelke, E., 2004. Core systems of number. Trends in Cognitive Sciences, 8(7), pp.307-314. Available at: http://linkinghub.elsevier.com/retrieve/pii/S1364661304001317.

Feigenson, L., Libertus, M.E. \& Halberda, J., 2013. Links Between the Intuitive Sense of Number and Formal Mathematics Ability. Child Development Perspectives, 7(2), pp.74-79.

Ferrari, M. \& Quaresima, V., 2012. A brief review on the history of human functional nearinfrared spectroscopy (fNIRS) development and fields of application. NeuroImage, 63(2), pp.921-935. Available at: http://www.sciencedirect.com/science/article/pii/S1053811912003308.

Fias, W. et al., 1996. The Importance of Magnitude Information in Numerical Processing: Evidence from the SNARC Effect. 1Mathematical Cognition, 2(1), pp.65-110.

Fias, W. et al., 2003. Parietal Representation of Symbolic and Nonsymbolic Magnitude. Journal of Cognitive Neuroscience, 15(1), pp.47-56. Available at: http://dx.doi.org/10.1162/089892903321107819. 
Fias, W., van Dijck, J.P. \& Gevers, W., 2011. How is Number Associated with Space? The Role of Working Memory. Space, Time and Number in the Brain, 1(c), pp.133-148. Available at: http://dx.doi.org/10.1016/B978-0-12-385948-8.00010-4.

Fischer, M.H. et al., 2003. Perceiving numbers causes spatial shifts of attention. Nature neuroscience, 6(6), pp.555-556.

Fischer, M.H., 2001. Number processing induces spatial performance biases. Neurology, 57(5), pp.822-826.

Fuson, K.C., 1988. Children's counting and concepts of number. Springer-Verlag, ed., New York: Springer-Verlag. Available at: http://psycnet.apa.org/psycinfo/1987-98811-000.

Gallistel, C.R. \& Gelman, I., 2000. Non-verbal numerical cognition: from reals to integers. Trends in cognitive sciences, 4(2), pp.59-65.

Gallistel, C.R. \& Gelman, R., 1992. Preverbal and verbal counting and computation. Cognition, 44(1-2), pp.43-74.

Geary, D.C. et al., 1987. Cognitive addition: Comparison of learning disabled and academically normal elementary school children. Cognitive Development, 2(3), pp.249_ 269. Available at: http://linkinghub.elsevier.com/retrieve/pii/S088520148790075X.

Geary, D.C. et al., 2008. Development of number line representations in children with mathematical learning disability. Developmental neuropsychology, 33(3), pp.277-299. Available at: http://dx.doi.org/10.1080/87565640801982361 [Accessed July 15, 2012].

Geary, D.C. et al., 2009. First-Grade Predictors of Mathematical Learning Disability: A Latent Class Trajectory Analysis. Cognitive development, 24(4), pp.411-429. Available at:

http://www.pubmedcentral.nih.gov/articlerender.fcgi?artid=2813681\&tool=pmcentrez\& rendertype $=$ abstract [Accessed July 20, 2012].

Geary, D.C., 2011. Cognitive predictors of achievement growth in mathematics: a 5-year longitudinal study. Developmental psychology, 47(6), pp.1539-52. Available at: 
http://www.ncbi.nlm.nih.gov/pubmed/21942667 [Accessed July 20, 2012].

Gebuis, T. \& Reynvoet, B., 2011. Generating nonsymbolic number stimuli. Behavior research methods, 43(4), pp.981-6. Available at: http://www.ncbi.nlm.nih.gov/pubmed/21512872 [Accessed November 6, 2012].

Gebuis, T. \& Reynvoet, B., 2012a. The interplay between nonsymbolic number and its continuous visual properties. Journal of experimental psychology. General, 141(4), pp.642-8. Available at: http://www.ncbi.nlm.nih.gov/pubmed/22082115 [Accessed December 17, 2014].

Gebuis, T. \& Reynvoet, B., 2012b. The role of visual information in numerosity estimation. PloS one, 7(5), p.e37426. Available at: http://www.pubmedcentral.nih.gov/articlerender.fcgi?artid=3355123\&tool=pmcentrez\& rendertype $=$ abstract $[$ Accessed December 17, 2014].

Gebuis, T. et al., 2009. Automatic quantity processing in 5-year olds and adults. Cognitive processing, 10(2), pp.133-42.

Gebuis, T., Gevers, W. \& Cohen Kadosh, R., 2014. Topographic representation of high-level cognition: numerosity or sensory processing? Trends in cognitive sciences, 18(1), pp.13. Available at: http://www.ncbi.nlm.nih.gov/pubmed/24129333.

Gelman, R. \& Gallistel, C.R., 1978. The Child's Understanding of Number, Cambridge, MA: Harvard University Press.

Gevers, W. et al., 2010. Verbal-spatial and visuospatial coding of number-space interactions. Journal of experimental psychology. General, 139(1), pp.180-190.

Gevers, W., Cohen Kadosh, R., \& Gebuis, T. (2016). The sensory integration theory: an alternative to the Approximate Number System. In A. Henik (Ed.), Continuous Issues in Numerical Cognition: Elsevier.

Gilmore, C. et al., 2013. Individual differences in inhibitory control, not non-verbal number acuity, correlate with mathematics achievement. C. Chambers, ed. PloS one, 8(6), 
p.e67374. Available at: http://dx.plos.org/10.1371/journal.pone.0067374 [Accessed January 24, 2014].

Girelli, L., Lucangeli, D. \& Butterworth, B., 2000. The development of automaticity in accessing number magnitude. Journal of experimental child psychology, 76(2), pp.104122.

Grabner, R.H. et al., 2009. To retrieve or to calculate? Left angular gyrus mediates the retrieval of arithmetic facts during problem solving. Neuropsychologia, 47(2), pp.604608.

Halberda, J. et al., 2012. Number sense across the lifespan as revealed by a massive Internetbased sample. Proceedings of the National Academy of Sciences of the United States of America, 109(28), pp.11116-20. Available at:

http://www.ncbi.nlm.nih.gov/pubmed/22733748 [Accessed July 13, 2012].

Halberda, J., 2011. What is a Weber fraction? in Press.

Halberda, J., Mazzocco, M.M.M. \& Feigenson, L., 2008. Individual differences in non-verbal number acuity correlate with maths achievement. Nature, 455(7213), pp.665-8.

Available at: http://www.ncbi.nlm.nih.gov/pubmed/18776888.

Hauser, T.U. et al., 2013. Enhancing performance in numerical magnitude processing and mental arithmetic using transcranial Direct Current Stimulation (tDCS). Frontiers in Human Neuroscience, 7, p.9. Available at: $<$ Go to ISI $>$ ://WOS:000319996200001.

Henik, a \& Tzelgov, J., 1982. Is three greater than five: the relation between physical and semantic size in comparison tasks. Memory \& cognition, 10(4), pp.389-395.

Hittmair-Delazer, M., Semenza, C. \& Denes, G., 1994. Concepts and facts in calculation. Brain : a journal of neurology, 117 ( Pt 4, pp.715-728.

Hoffmann, D. et al., 2013. Developing number-space associations: SNARC effects using a color discrimination task in 5-year-olds. Journal of Experimental Child Psychology, 116(4), pp.775-791. Available at: http://dx.doi.org/10.1016/j.jecp.2013.07.013. 
Holloway, I.D. \& Ansari, D., 2009. Mapping numerical magnitudes onto symbols: the numerical distance effect and individual differences in children's mathematics achievement. Journal of experimental child psychology, 103(1), pp.17-29. Available at: http://www.ncbi.nlm.nih.gov/pubmed/18513738 [Accessed May 5, 2014].

Hurst, M. et al., 2014. 123s and ABCs: developmental shifts in logarithmic-to-linear responding reflect fluency with sequence values. Developmental science, pp.1-13. Available at: http://www.ncbi.nlm.nih.gov/pubmed/24636167 [Accessed April 29, 2014].

Hyde, D. C. (2011). Two systems of non-symbolic numerical cognition. Frontiers in Human Neuroscience, 5. doi:10.3389/fnhum.2011.00150

Hyde, D.C. \& Spelke, E.S., 2009. All numbers are not equal: an electrophysiological investigation of small and large number representations. Journal of cognitive neuroscience, 21(6), pp.1039-53.

Hyde, D.C. \& Spelke, E.S., 2011. Neural signatures of number processing in human infants: evidence for two core systems underlying numerical cognition. Developmental science, 14(2), pp.360-71. Available at: http://www.pubmedcentral.nih.gov/articlerender.fcgi? artid=3050652\&tool=pmcentrez\& rendertype $=$ abstract [Accessed August 19, 2013].

Hyde, D.C. et al., 2010. Near-infrared spectroscopy shows right parietal specialization for number in pre-verbal infants. NeuroImage, 53(2), pp.647-652.

Hyde, D.C., 2011. Two systems of non-symbolic numerical cognition. Frontiers in Human Neuroscience, 5. Available at: http://www.frontiersin.org/Journal/Abstract.aspx?s=537\&name=human_neuroscience\& ART_DOI=10.3389/fnhum.2011.00150.

Imbo, I., Duverne, S. \& Lemaire, P., 2007. Working memory, strategy execution, and strategy selection in mental arithmetic. Quarterly journal of experimental psychology 
(2006), 60(9), pp.1246-64. Available at:

http://www.ncbi.nlm.nih.gov/pubmed/17676556.

Inglis, M. \& Gilmore, C., 2014. Indexing the approximate number system. Acta Psychologica, 145(1), pp.147-155. Available at:

http://dx.doi.org/10.1016/j.actpsy.2013.11.009 [Accessed January 21, 2014].

Ischebeck, A. et al., 2009. Flexible transfer of knowledge in mental arithmetic - An fMRI study. NeuroImage, 44(3), pp.1103-1112. Available at:

http://linkinghub.elsevier.com/retrieve/pii/S105381190801118X.

Iuculano, T. \& Cohen Kadosh, R., 2013. The mental cost of cognitive enhancement. The Journal of neuroscience : the official journal of the Society for Neuroscience, 33(10), pp.4482-6. Available at:

http://www.pubmedcentral.nih.gov/articlerender.fcgi? artid=3672974\&tool=pmcentrez\& rendertype $=$ abstract [Accessed July 30, 2014].

Izard, V. \& Dehaene, S., 2008. Calibrating the mental number line. Cognition, 106(3), pp.1221-47. Available at: http://www.ncbi.nlm.nih.gov/pubmed/17678639 [Accessed January 23, 2014].

Izard, V. et al., 2009. Newborn infants perceive abstract numbers. Proceedings of the National Academy of Sciences of the United States of America, 106(25), pp.10382-5.

Izard, V., Dehaene-Lambertz, G. \& Dehaene, S., 2008. Distinct Cerebral Pathways for Object Identity and Number in Human Infants. PLoS Biol, 6(2), p.e11. Available at: http://dx.doi.org/10.1371\%2Fjournal.pbio.0060011.

Javadi, A.H. et al., 2014. Transcranial electrical brain stimulation modulates neuronal tuning curves in perception of numerosity and duration. NeuroImage, 102, pp.451-457.

Johnson, M.H., 2001. Functional brain development in humans. Nature reviews. Neuroscience, 2(7), pp.475-483.

Karmiloff-Smith, A., 1998. Development itself is the key to understanding developmental 
disorders. Trends Cogn Sci, 2(10), pp.389-398. Available at:

http://www.sciencedirect.com/science/article/pii/S1364661398012303.

Kaufman, E.L. et al., 1949. The Discrimination of Visual Number. The American journal of psychology, 62(4), pp.498-525.

Kaufmann, L. et al., 2011. Meta-analyses of developmental fMRI studies investigating typical and atypical trajectories of number processing and calculation. Developmental neuropsychology, 36, pp.763-787.

Kaufmann, L., Wood, G., Rubinsten, O., \& Henik, A. (2011). Meta-Analyses of Developmental fMRI Studies Investigating Typical and Atypical Trajectories of Number Processing and Calculation. Developmental Neuropsychology, 36(6), 763-787. doi:10.1080/87565641.2010.549884

Klahr, D. \& Wallace, J.G., 1973. The role of quantification operators in the development of conservation of quantity. Cognitive Psychology, 4(3), pp.301-327.

Knops, A., Dehaene, S., et al., 2014. Can approximate mental calculation account for operational momentum in addition and subtraction? Quarterly journal of experimental psychology (2006), 67(September), pp.37-41. Available at:

http://www.ncbi.nlm.nih.gov/pubmed/24499435 [Accessed July 15, 2014].

Knops, A., Piazza, M., et al., 2014. A Shared, Flexible Neural Map Architecture Reflects Capacity Limits in Both Visual Short-Term Memory and Enumeration. Journal of Neuroscience, 34(30), pp.9857-9866. Available at:

http://www.jneurosci.org/cgi/doi/10.1523/JNEUROSCI.2758-13.2014 [Accessed July $23,2014]$.

Koechlin, E., 1997. Numerical Transformations in Five-month-old Human Infants. Mathematical Cognition, 3(2), pp.89-104.

Koponen, T. et al., 2007. Cognitive predictors of single-digit and procedural calculation skills and their covariation with reading skill. Journal of Experimental Child Psychology, 
97(3), pp.220-241.

Krause, B. \& Cohen Kadosh, R., 2013. Can transcranial electrical stimulation improve learning difficulties in atypical brain development? A future possibility for cognitive training. Developmental Cognitive Neuroscience, 6, pp.176-194. Available at: http://www.pubmedcentral.nih.gov/articlerender.fcgi?artid=4064117\&tool=pmcentrez\& rendertype $=$ abstract [Accessed July 25, 2014].

Krekelberg, B., Boynton, G.M. \& van Wezel, R.J., 2006. Adaptation: from single cells to BOLD signals. Trends Neurosci, 29(5), pp.250-256.

Kriegeskorte, N., Mur, M., \& Bandettini, P. A. (2008). Representational similarity analysisconnecting the branches of systems neuroscience. Frontiers in systems neuroscience, 2, 4.

Kroesbergen, E.H. et al., 2009. Individual Differences in Early Numeracy: the Role of Executive Functions and Subitizing. Journal of Psychoeducational Assessment, 27(3), pp.226-236.

Kucian, K. et al., 2011. Mental number line training in children with developmental dyscalculia. NeuroImage, 57(3), pp.782-95.

Landerl, K., 2013. Development of numerical processing in children with typical and dyscalculic arithmetic skills-a longitudinal study. Frontiers in psychology, 4(July), p.459.

Landerl, K., Bevan, A. \& Butterworth, B., 2004. Developmental dyscalculia and basic numerical capacities: a study of 8-9-year-old students. Cognition, 93(2), pp.99-125.

Le Corre, M. \& Carey, S., 2007. One, two, three, four, nothing more: an investigation of the conceptual sources of the verbal counting principles. Cognition, 105(2), pp.395-438.

Le Corre, M. et al., 2006. Re-visiting the competence/performance debate in the acquisition of the counting principles. Cognitive psychology, 52(2), pp.130-69.

Le Corre, M., 2014. Children acquire the later-greater principle after the cardinal principle. 
British Journal of Developmental Psychology, 32(2), pp.163-177.

Lee, K. et al., 2004. Working memory and literacy as predictors of performance on algebraic word problems. Journal of Experimental Child Psychology, 89(2), pp.140-158.

Lemaire, P., 1996. The Role of Working Memory Resources in Simple Cognitive Arithmetic. European Journal of Cognitive Psychology, 8(1), pp.73-104.

Libertus, M.E., Feigenson, L. \& Halberda, J., 2013. Is Approximate Number Precision a Stable Predictor of Math Ability? Learning and individual differences, 25, pp.126-133. Available at: http://www.sciencedirect.com/science/article/pii/S1041608013000198 [Accessed September 20, 2013].

Logie, R.H., Gilhooly, K.J. \& Wynn, V., 1994. Counting on working memory in arithmetic problem solving. Memory \& cognition, 22(4), pp.395-410.

Lucangeli, D. et al., 2003. Effective strategies for mental and written arithmetic calculation from the third to the fifth grade. Educational Psychology, 23(5), pp.507-520.

Lyons, I.M. et al., 2014. Numerical predictors of arithmetic success in grades 1-6. Developmental Science, 5, pp.714-726.

Lyons, I.M., Ansari, D. \& Beilock, S.L., 2015. Qualitatively different coding of symbolic and nonsymbolic numbers in the human brain. Hum Brain Mapp, 36(2), pp.475-488.

Mammarella, I.C., Lucangeli, D. \& Cornoldi, C., 2010. Spatial Working Memory and Arithmetic Deficits in Children With Nonverbal Learning Difficulties. Journal of Learning Disabilities, 43(5), pp.455-468. Available at: http://ldx.sagepub.com/cgi/doi/10.1177/0022219409355482.

Mandler, G. \& Shebo, B.J., 1982. Subitizing : An Analysis of Its Component Processes. Journal of experimental psychology. General, 111(1), pp.1-22.

Marangolo, P., Nasti, M. \& Zorzi, M., 2004. Selective impairment for reading numbers and number words: a single case study. Neuropsychologia, 42(8), pp.997-1006. Available at: http://www.sciencedirect.com/science/article/pii/S0028393204000120. 
Mazza, V. \& Caramazza, A., 2015. Multiple object individuation and subitizing in enumeration: a view from electrophysiology. Frontiers in Human Neuroscience, 9, p.162. Available at: http://www.ncbi.nlm.nih.gov/pmc/articles/PMC4382968/.

Mazzocco, M.M.M., Feigenson, L. \& Halberda, J., 2011a. Impaired acuity of the approximate number system underlies mathematical learning disability (dyscalculia). Child development, 82(4), pp.1224-37.

Mazzocco, M.M.M., Feigenson, L. \& Halberda, J., 2011b. Preschoolers' precision of the approximate number system predicts later school mathematics performance. PloS one, 6(9), p.e23749.

McCloskey, M., Caramazza, A. \& Basili, A., 1985. Cognitive mechanisms in number processing and calculation: evidence from dyscalculia. Brain Cogn, 4(2), pp.171-196. McCrink, K. \& Birdsall, W., 2015. Numerical Abilities and Arithmetic in Infancy. In The Oxford Handbook of Numerical Cognition. p. 258.

Mccrink, K. \& Wynn, K., 2004. Large-Number Addition and Subtraction by 9-Month-Old Infants.

McCrink, K. \& Wynn, K., 2007. Ratio abstraction by 6-month-old infants. Psychological science, 18(8), pp.740-5. Available at: http://www.ncbi.nlm.nih.gov/pubmed/17680947.

McCrink, K. \& Wynn, K., 2009. Operational momentum in large-number addition and subtraction by 9-month-olds. Journal of experimental child psychology, 103(4), pp.4008. Available at: http://www.ncbi.nlm.nih.gov/pubmed/19285683 [Accessed September $8,2014]$.

McCrink, K., Dehaene, S. \& Dehaene-Lambertz, G., 2007. Moving along the number line: operational momentum in nonsymbolic arithmetic. Perception \& psychophysics, 69(8), pp.1324-33. Available at: http://www.ncbi.nlm.nih.gov/pubmed/18078224.

McLean, J.F. \& Hitch, G.J., 1999. Working memory impairments in children with specific arithmetic learning difficulties. Journal of experimental child psychology, 74(3), 
pp. 240-60.

Moeller, K. et al., 2009. Children's early mental number line: logarithmic or decomposed linear? Journal of experimental child psychology, 103(4), pp.503-15.

Moeller, K. et al., 2011. Effects of finger counting on numerical development the opposing views of neurocognition and mathematics education. Frontiers in Psychology, 2(NOV), pp.1-5.

Moeller, K., Fischer, U., et al., 2015. Computers in mathematics education - Training the mental number line. Computers in Human Behavior, 48, pp.597-607. Available at: http://linkinghub.elsevier.com/retrieve/pii/S074756321500062X.

Moeller, K., Willmes - von Hinckeldey, K. F., \& Klein, E. (2015). A review on functional and structural brain connectivity in numerical cognition. Frontiers in Human Neuroscience, 9.

Moeller, K., Willmes - von Hinckeldey, K.F. \& Klein, E., 2015. A review on functional and structural brain connectivity in numerical cognition. Frontiers in Human Neuroscience, 9. Available at:

http://www.frontiersin.org/Journal/Abstract.aspx?s=537\&name=human_neuroscience\& ART_DOI=10.3389/fnhum.2015.00227.

Molko, N. et al., 2003. Functional and Structural Alterations of the Intraparietal Sulcus in a Developmental Dyscalculia of Genetic Origin. Neuron, 40(4), pp.847-858. Available at: http://www.sciencedirect.com/science/article/pii/S0896627303006706.

Moyer, R.S. \& Landauer, T.K., 1967. Time required for judgements of numerical inequality. Nature, 215(5109), pp.1519-1520.

Mussolin, C., Mejias, S. \& Noël, M.-P., 2010. Symbolic and nonsymbolic number comparison in children with and without dyscalculia. Cognition, 115(1), pp.10-25. Available at: http://www.ncbi.nlm.nih.gov/pubmed/20149355 [Accessed February 4, 2014]. 
Nieder, A. \& Miller, E.K., 2004. A parieto-frontal network for visual numerical information in the monkey. Proc Natl Acad Sci U S A, 101(19), pp.7457-7462.

Nieder, A. (2013). Coding of abstract quantity by 'number neurons' of the primate brain. J Comp Physiol A Neuroethol Sens Neural Behav Physiol, 199(1), 1-16. doi:10.1007/s00359-012-0763-9

Nieder, A., \& Miller, E. K. (2004). A parieto-frontal network for visual numerical information in the monkey. Proceedings of the National Academy of Sciences of the United States of America, 101(19), 7457-7462.

Nieder, A., 2013. Coding of abstract quantity by "number neurons" of the primate brain. J Comp Physiol A Neuroethol Sens Neural Behav Physiol, 199(1), pp.1-16.

Nieder, A., Freedman, D.J. \& Miller, E.K., 2002. Representation of the quantity of visual items in the primate prefrontal cortex. Science (New York, N.Y.), 297(5587), pp.170811. Available at: http://www.ncbi.nlm.nih.gov/pubmed/12215649.

Nuerk, H.C., Wood, G. \& Willmes, K., 2005. The universal SNARC effect: The association between number magnitude and space is amodal. Experimental Psychology, 52(3), pp.187-194.

Oakes, L.M., 2011. Using Habituation of Looking Time to Assess Mental Processes in Infancy. Journal of Cognition and Development, 11(3), pp.1-10.

Opfer, J.E. \& Thompson, C. a, 2006. Even Early Representations of Numerical Magnitude are Spatially Organized: Evidence for a Directional Magnitude Bias in Pre-Reading Preschoolers. Proceedings of the XXVIII Annual Conference of the Cognitive Science Societe, (1), pp.639-644.

Passolunghi, M.C. \& Cornoldi, C., 2008. Working memory failures in children with arithmetical difficulties. Child neuropsychology : a journal on normal and abnormal development in childhood and adolescence, 14(December), pp.387-400.

Passolunghi, M.C. \& Siegel, L.S., 2001. Short-Term Memory, Working Memory, and 
Inhibitory Control in Children with Difficulties in Arithmetic Problem Solving. Journal of Experimental Child Psychology, 80(1), pp.44-57. Available at: http://linkinghub.elsevier.com/retrieve/pii/S0022096500926262.

Passolunghi, M.C., Cargnelutti, E. \& Pastore, M., 2014. The contribution of general cognitive abilities and approximate number system to early mathematics. The British journal of educational psychology. Available at: http://www.ncbi.nlm.nih.gov/pubmed/25175790 [Accessed September 2, 2014].

Patro, K. \& Haman, M., 2012. The spatial-numerical congruity effect in preschoolers. Journal of experimental child psychology, 111(3), pp.534-42. Available at: http://www.ncbi.nlm.nih.gov/pubmed/22153910 [Accessed February 5, 2014].

Piazza, M. \& Eger, E., 2015. Neural foundations and functional specificity of number representations. Neuropsychologia.

Piazza, M. et al., 2002. Are subitizing and counting implemented as separate or functionally overlapping processes? NeuroImage, 15(2), pp.435-46.

Piazza, M. et al., 2004. Tuning curves for approximate numerosity in the human intraparietal sulcus. Neuron, 44(3), pp.547-55.

Piazza, M. et al., 2007. A magnitude code common to numerosities and number symbols in human intraparietal cortex. Neuron, 53(2), pp.293-305. Available at: http://www.ncbi.nlm.nih.gov/pubmed/17224409.

Piazza, M. et al., 2010. Developmental trajectory of number acuity reveals a severe impairment in developmental dyscalculia. Cognition, 116(1), pp.33-41. Available at: http://www.ncbi.nlm.nih.gov/pubmed/20381023 [Accessed November 6, 2010].

Piazza, M. et al., 2011. Subitizing reflects visuo-spatial object individuation capacity. Cognition, 121(1), pp.147-53. Available at: http://www.ncbi.nlm.nih.gov/pubmed/21679934 [Accessed November 18, 2012].

Piazza, M., 2010. Neurocognitive start-up tools for symbolic number representations. Trends 
in cognitive sciences, 14(12), pp.542-51. Available at:

http://www.ncbi.nlm.nih.gov/pubmed/21055996 [Accessed July 15, 2012].

Pinhas, M. \& Fischer, M.H., 2008. Mental movements without magnitude? A study of spatial biases in symbolic arithmetic. Cognition, 109(3), pp.408-415.

Piras, F. \& Marangolo, P., 2009. Word and number reading in the brain: Evidence from a Voxel-based Lesion-symptom Mapping study. Neuropsychologia, 47(8-9), pp.19441953. Available at:

http://www.sciencedirect.com/science/article/pii/S0028393209001274.

Platt, J.R. \& Johnson, D.M., 1971. Localization of position within a homogeneous behavior chain: Effects of error contingencies. Learning and Motivation, 2, pp.386-414.

Popescu, T. et al., 2016. Transcranial random noise stimulation mitigates increased difficulty in an arithmetic learning task. Neuropsychologia, 81, pp.255-264. Available at: http://www.sciencedirect.com/science/article/pii/S0028393215302682.

Prado, J. et al., 2011. Distinct representations of subtraction and multiplication in the neural systems for numerosity and language. Human Brain Mapping, 32(11), pp.1932-1947. Available at: http://dx.doi.org/10.1002/hbm.21159.

Prado, J., Mutreja, R. \& Booth, J.R., 2014. Developmental dissociation in the neural responses to simple multiplication and subtraction problems. Developmental science, 17(4), pp.537-552. Available at: http://dx.doi.org/10.1111/desc.12140.

Price, G.R. et al., 2012. Nonsymbolic numerical magnitude comparison: reliability and validity of different task variants and outcome measures, and their relationship to arithmetic achievement in adults. Acta psychologica, 140(1), pp.50-57. Available at: http://www.ncbi.nlm.nih.gov/pubmed/22445770 [Accessed November 8, 2012].

Proctor, R.W. \& Cho, Y.S., 2006. Polarity correspondence: A general principle for performance of speeded binary classification tasks. Psychological bulletin, 132(3), pp.416-442. 
Pylyshyn, Z.W., 2001. Visual indexes, preconceptual objects, and situated vision. Cognition, 80(1-2), pp.127-58.

Ramani, G.B., Siegler, R.S. \& Hitti, A., 2012. Taking it to the classroom: Number board games as a small group learning activity. Journal of Educational Psychology, 104(3), pp.661-672.

Reeve, R. et al., 2012. Stability and change in markers of core numerical competencies. Journal of experimental psychology. General, 141(4), pp.649-66.

Reynvoet, B. \& Brysbaert, M., 1999. Single-digit and two-digit Arabic numerals address the same semantic number line. Cognition, 72(2), pp.191-201.

Reynvoet, B. \& Brysbaert, M., 2004. Cross-Notation Number Priming Investigated at Different Stimulus Onset Asynchronies in Parity and Naming Tasks. Experimental Psychology, 51(2), pp.81-90.

Reynvoet, B., Caessens, B. \& Brysbaert, M., 2002. Automatic stimulus-response associations may be semantically mediated. Psychonomic bulletin \& review, 9(1), pp.107-112.

Rosca, E.C., 2009. A case of acalculia due to impaired procedural knowledge. Neurol Sci, 30(2), pp.163-170.

Rousselle, L. \& Noël, M.-P., 2007. Basic numerical skills in children with mathematics learning disabilities: a comparison of symbolic vs non-symbolic number magnitude processing. Cognition, 102(3), pp.361-95.

Rubinsten, O., Henik, A., Berger, A., \& Shahar-Shalev, S. (2002). The development of internal representations of magnitude and their association with Arabic numerals. J Exp Child Psychol, 81(1), 74-92. doi:10.1006/jecp.2001.2645

Rykhlevskaia, E. et al., 2009. Neuroanatomical correlates of developmental dyscalculia: combined evidence from morphometry and tractography. Frontiers in Human Neuroscience, 3. Available at: http://www.frontiersin.org/Journal/Abstract.aspx?s=537\&name=human_neuroscience\& 
ART_DOI=10.3389/neuro.09.051.2009.

Santens, S. \& Gevers, W., 2008. The SNARC effect does not imply a mental number line. Cognition, 108(1), pp.263-270.

Sapountzis, P. et al., 2010. A comparison of fMRI adaptation and multivariate pattern classification analysis in visual cortex. NeuroImage, 49(2), pp.1632-1640. Available at: http://www.ncbi.nlm.nih.gov/pmc/articles/PMC2793370/.

Sarkar, A. \& Cohen Kadosh, R., 2016. Chapter 10 - Transcranial Electrical Stimulation and the Enhancement of Numerical Cognition. In D. B. B. C. G. M. Koepke, ed. Development of Mathematical Cognition. San Diego: Academic Press, pp. 245-296. Available at: http://www.sciencedirect.com/science/article/pii/B9780128018712000101.

Sarkar, A., Dowker, A. \& Kadosh, R.C., 2014. Cognitive Enhancement or Cognitive Cost : Trait-Specific Outcomes of Brain Stimulation in the Case of Mathematics Anxiety. , 34(50), pp.16605-16610.

Sarnecka, B.W. \& Carey, S., 2008. How counting represents number: what children must learn and when they learn it. Cognition, 108(3), pp.662-74. Available at: http://www.ncbi.nlm.nih.gov/pubmed/18572155 [Accessed May 23, 2013].

Sarnecka, B.W. et al., 2007. From grammatical number to exact numbers: early meanings of "one", "two", and "three" in English, Russian, and Japanese. Cognitive psychology, 55(2), pp.136-68. Available at:

http://www.pubmedcentral.nih.gov/articlerender.fcgi?artid=2322941\&tool=pmcentrez\& rendertype=abstract [Accessed July 17, 2014].

Sasanguie, D. et al., 2013. Approximate number sense, symbolic number processing, or number-space mappings: what underlies mathematics achievement? Journal of experimental child psychology, 114(3), pp.418-31. Available at: http://www.ncbi.nlm.nih.gov/pubmed/23270796 [Accessed January 26, 2014]. Schleifer, P. \& Landerl, K., 2011. Subitizing and counting in typical and atypical 
development. Developmental Science, 14(2), pp.280-291.

Schwarz, W. \& Heinze, H.J., 1998. On the interaction of numerical and size information in digit comparison: A behavioral and event-related potential study. Neuropsychologia, 36(11), pp.1167-1179.

Sella, F. et al., 2012. Strategy Selection in ADHD Characteristics Children: A Study in Arithmetic. Journal of attention disorders. Available at: http://www.ncbi.nlm.nih.gov/pubmed/22451509 [Accessed April 12, 2012].

Sella, F. et al., 2014. Number line estimation in children with developmental dyscalculia. Learning disabilties: A Contemporary Journal, 11(2), pp.41-49.

Sella, F. et al., 2015a. Spontaneous non-verbal counting in toddlers. Developmental science, pp.1-9.

Sella, F. et al., 2015b. Varieties of Quantity Estimation in Children. Developmental psychology, 51(6), pp.758-770.

Sella, F. et al., 2016. Basic and advanced numerical performances relate to mathematical expertise but are fully mediated by visuospatial skills. Journal of Experimental Psychology: Learning, Memory, and Cognition, 42(April), pp.1458-1472.

Sella, F., Lanfranchi, S. \& Zorzi, M., 2013. Enumeration skills in Down syndrome. Research in Developmental Disabilities, 34(11), pp.3798-3806. Available at: http://dx.doi.org/10.1016/j.ridd.2013.07.038 [Accessed September 8, 2013].

Shaki, S. \& Fischer, M.H., 2008. Reading space into numbers - a cross-linguistic comparison of the SNARC effect. Cognition, 108(2), pp.590-599.

Shaki, S., Fischer, M.H. \& Petrusic, W.M., 2009. Reading habits for both words and numbers contribute to the SNARC effect. Psychonomic bulletin \& review, 16(2), pp.328-331.

Sharon, T. \& Wynn, K., 1998. INDIVIDUATION OF ACTIONS FROM CONTINUOUS MOTION. Psychological science, 9(5), pp.357-362.

Siegler, R.S. \& Booth, J.L., 2004. Development of numerical estimation in young children. 
Child development, 75(2), pp.428-44. Available at:

http://www.ncbi.nlm.nih.gov/pubmed/15056197.

Siegler, R.S. \& Opfer, J.E., 2003. The development of numerical estimation: evidence for multiple representations of numerical quantity. Psychological Science, 14(3), pp.23743.

Siegler, R.S. \& Ramani, G.B., 2008. Playing linear numerical board games promotes lowincome children's numerical development. Developmental science, 11(5), pp.655-61.

Siegler, R.S. \& Ramani, G.B., 2009. Playing linear number board games—but not circular ones - improves low-income preschoolers' numerical understanding. Journal of Educational Psychology, 101(3), pp.545-560. Available at: http://doi.apa.org/getdoi.cfm?doi=10.1037/a0014239.

Simon, T.J., Hespos, S.J. \& Rochat, P., 1995. Do infants understand simple arithmetic? A replication of Wynn (1992). Cognitive Development, 10(2), pp.253-269.

Sirois, S. \& Mareschal, D., 2002. Models of habituation in infancy. Trends in Cognitive Sciences, 6(7), pp.293-298.

Slusser, E.B., Santiago, R.T. \& Barth, H.C., 2013. Developmental change in numerical estimation. Journal of experimental psychology. General, 142(1), pp.193-208.

Snowball, A. et al., 2013. Long-term enhancement of brain function and cognition using cognitive training and brain stimulation. Current biology : CB, 23(11), pp.987-92. Available at:

http://www.pubmedcentral.nih.gov/articlerender.fcgi? artid=3675670\&tool=pmcentrez\& rendertype $=$ abstract [Accessed July 14, 2014].

Soltész, F., Szücs, D. \& Szucs, L., 2010. Relationships between magnitude representation, counting and memory in 4- to 7-year-old children: a developmental study. Behavioral and brain functions : BBF, 6, p.13. Available at:

http://www.pubmedcentral.nih.gov/articlerender.fcgi?artid=2833140\&tool=pmcentrez\& 
rendertype $=$ abstract.

Spelke, E.S., 2000. Core Knowledge of Objects. American Psychologist, (November), pp.1233-1243.

Spinath, B. et al., 2006. Predicting school achievement from general cognitive ability, selfperceived ability, and intrinsic value. Intelligence, 34(4), pp.363-374.

Starkey, P. \& Cooper, R.G., 1980. Perception of Numbers by Human Infants. Scien, 210(4473), pp.1033-1035.

Starkey, P., Spelke, E. \& Gelman, R., 1983. Detection of intermodal numerical correspondence by human infants. Science, 222, pp.179-181.

Starr, A., Libertus, M.E. \& Brannon, E.M., 2013. Number sense in infancy predicts mathematical abilities in childhood. Proceedings of the National Academy of Sciences, 110(45), pp.1-5. Available at: http://www.pnas.org/content/110/45/18116.full [Accessed October 22, 2013].

Stoianov, I. \& Zorzi, M., 2012. Emergence of a "visual number sense" in hierarchical generative models. Nature neuroscience, 15(2), pp.194-196.

Strauss, M.S. \& Curtis, L.E., 1981. Infant perception of numerosity. Child development, 52(4), pp.1146-1152.

Sullivan, J. \& Barner, D., 2014. Inference and Association in Children's Early Numerical Estimation. Child development, pp.1-16. Available at: http://www.ncbi.nlm.nih.gov/pubmed/24397891 [Accessed February 8, 2014].

Szücs, D. et al., 2013. Developmental dyscalculia is related to visuo-spatial memory and inhibition impairment. Cortex; a journal devoted to the study of the nervous system and behavior, 49(10), pp.2674-88. Available at: http://www.pubmedcentral.nih.gov/articlerender.fcgi? artid=3878850\&tool=pmcentrez $\&$ rendertype $=$ abstract [Accessed January 23, 2014].

Szűcs, D. et al., 2014. Cognitive components of a mathematical processing network in 9- 
year-old children. Developmental Science, 17(4), pp.506-524. Available at:

http://doi.wiley.com/10.1111/desc.12144 [Accessed February 27, 2014].

Thioux, M. et al., 1998. The Isolation of Numerals at the Semantic Level. Neurocase, 4(4-5), pp.371-389. Available at: http://dx.doi.org/10.1080/13554799808410633.

Thompson, I., 1999. Mental Calculation Strategies for Addition and Subtraction. Mathematics in School, 28(5), pp.2-4.

Thompson, I., 2000. Mental calculation strategies for addition and subtraction: part 2. Mathematics in School, 29(5), pp.24-26.

Threlfall, J., 2000. Mental Calculation Strategies. Research in Mathematics Education, 2(1), pp.77-90. Available at:

http://www.tandfonline.com/doi/abs/10.1080/14794800008520069.

Treiber, F. \& Wilcox, S., 1984. Discrimination of number by infants. Infant Behavior and Development, 7(1), pp.93-100.

Trick, L.M. \& Pylyshyn, Z.W., 1994. Why Are Small and Large Numbers Enumerated Differently? A Limited-Capacity Preattentive Stage in Vision. Psychological Review, 101(1), pp.80-102.

Tschentscher, N. \& Hauk, O., 2014. How are things adding up? Neural differences between arithmetic operations are due to general problem solving strategies. NeuroImage, 92, pp.369-380. Available at:

http://www.sciencedirect.com/science/article/pii/S1053811914000925.

Tudusciuc, O. \& Nieder, A., 2007. Neuronal population coding of continuous and discrete quantity in the primate posterior parietal cortex. Proc Natl Acad Sci U S A, 104(36), pp.14513-14518.

Tzelgov, J., Meyer, J. \& Henik, A., 1992. Automatic and intentional processing of numerical information. Journal of Experimental Psychology: Learning, Memory, and Cognition, 18(1), pp.166-179. Available at: http://doi.apa.org/getdoi.cfm?doi=10.1037/0278- 
7393.18.1.166.

Vallence, A.M. \& Ridding, M.C., 2014. Non-invasive induction of plasticity in the human cortex: Uses and limitations. Cortex, 58, pp.261-271.

van Dijck, J.P. et al., 2012. The Heterogeneous Nature of Number-Space Interactions. Frontiers in Human Neuroscience, 5(January), pp.1-13.

van Dijck, J.P., Gevers, W. \& Fias, W., 2009. Numbers are associated with different types of spatial information depending on the task. Cognition, 113(2), pp.248-253. Available at: http://dx.doi.org/10.1016/j.cognition.2009.08.005.

van Galen, M.S. \& Reitsma, P., 2008. Developing access to number magnitude: A study of the SNARC effect in 7- to 9-year-olds. Journal of Experimental Child Psychology, 101(2), pp.99-113.

Van Loosbroek, E. \& Smitsman, A.W., 1990. Visual perception of numerosity in infancy. Developmental Psychology, 26(6), pp.916-922.

Verguts, T., Fias, W. \& Stevens, M., 2005. A model of exact small-number representation. Psychonomic bulletin \& review, 12(1), pp.66-80. Available at: http://www.ncbi.nlm.nih.gov/pubmed/15945201.

Vuokko, E., Niemivirta, M. \& Helenius, P., 2013. Cortical activation patterns during subitizing and counting. Brain Research, 1497, pp.40-52. Available at: http://www.sciencedirect.com/science/article/pii/S0006899312019300.

Walsh, V., 2003. A theory of magnitude: common cortical metrics of time, space and quantity. Trends in Cognitive Sciences, 7(11), pp.483-488. Available at: http://linkinghub.elsevier.com/retrieve/pii/S1364661303002304 [Accessed July 18, 2012].

Warrington, E.K., 1982. The fractionation of arithmetical skills: A single case study. The Quarterly Journal of Experimental Psychology Section A, 34(1), pp.31-51. Available at: http://dx.doi.org/10.1080/14640748208400856. 
Whalen, J. et al., 2002. Representing arithmetic table facts in memory: Evidence from acquired impairments. Cognitive neuropsychology, 19(6), pp.505-522.

Whalen, J., Gallistel, C.R. \& Gelman, R., 1999. Nonverbal Counting in Humans: The Psychophysics of Number Representation. Psychological Science, 10(2), pp.130-137.

Whalen, J., Gallistel, C.R. \& Gelman, R., 1999. Nonverbal Counting in Humans: The Psychophysics of Number Representation. Psychological Science, 10(2), pp.130-137.

Wood, J.N. \& Spelke, E.S., 2005. Infants' enumeration of actions: numerical discrimination and its signature limits. Developmental science, 8(2), pp.173-81. Available at: http://www.ncbi.nlm.nih.gov/pubmed/15720375.

Wynn, K., 1990. Children's understanding of counting. Cognition, 36, pp.155-193.

Wynn, K., 1992a. Addition and subtraction by human infants. Nature, 358, pp.749-750.

Wynn, K., 1992b. Children's Acquisition of the Number Words and the Counting System. Cognitive psychology, 24(24), pp.220-251.

Wynn, K., Bloom, P. \& Chiang, W.C., 2002. Enumeration of collective entities by 5-monthold infants. Cognition, 83(3).

Xu, F. \& Spelke, E.S., 2000. Large number discrimination in 6-month-old infants. Cognition, 74(1), pp.B1-B11.

Xu, F., Spelke, E.S. \& Goddard, S., 2005. Number sense in human infants. Developmental science, 8(1), pp.88-101. Available at: http://www.ncbi.nlm.nih.gov/pubmed/15647069.

Zhou, X. et al., 2007. Dissociated brain organization for single-digit addition and multiplication. NeuroImage, 35(2), pp.871-880. Available at: http://www.sciencedirect.com/science/article/pii/S1053811906012110.

Zorzi, M. \& Butterworth, B., 1999. A computational model of number comparison. In M. Hahn \& S. Stoness, eds. Twenty First Annual Meeting of the Cognitive Science Society. Mahwah, NJ.: Mahwah, p. 1.

Zorzi, M., Priftis, K. \& Umiltà, C., 2002. Neglect disrupts the mental number line. Nature, 
417(May), pp.138-140. 